\title{
Primordial Magnetic Field Effects on the CMB and Large-Scale Structure
}

\author{
Dai G. Yamazaki, ${ }^{1}$ Kiyotomo Ichiki, ${ }^{2}$ Toshitaka Kajino,, ${ }^{3,4}$ and Grant J. Mathews ${ }^{5}$ \\ ${ }^{1}$ Institute of Astronomy and Astrophysics, Academia Sinica, National Taiwan University, $7 F$ of Condensed Matter Sciences and Physics \\ Department Building, No.1, Roosevelt Road, Section 4 Taipei 10617, Taiwan \\ ${ }^{2}$ Department of Physics and Astrophysics, Nagoya University, Nagoya 464-8602, Japan \\ ${ }^{3}$ Theory Division, National Astronomical Observatory of Japan, Mitaka, Tokyo 181-8588, Japan \\ ${ }^{4}$ Department of Astronomy, Graduate School of Science, The University of Tokyo, Hongo 7-3-1, Bunkyo-ku, Tokyo 113-0033, Japan \\ ${ }^{5}$ Center for Astrophysics, Department of Physics, University of Notre Dame, Notre Dame, IN 46556, USA
}

Correspondence should be addressed to Dai G. Yamazaki, yamazaki@asiaa.sinica.edu.tw

Received 18 June 2010; Accepted 3 December 2010

Academic Editor: David Merritt

Copyright ( 92010 Dai G. Yamazaki et al. This is an open access article distributed under the Creative Commons Attribution License, which permits unrestricted use, distribution, and reproduction in any medium, provided the original work is properly cited.

\begin{abstract}
Magnetic fields are everywhere in nature, and they play an important role in every astronomical environment which involves the formation of plasma and currents. It is natural therefore to suppose that magnetic fields could be present in the turbulent high-temperature environment of the big bang. Such a primordial magnetic field (PMF) would be expected to manifest itself in the cosmic microwave background (CMB) temperature and polarization anisotropies, and also in the formation of large-scale structure. In this paper, we summarize the theoretical framework which we have developed to calculate the PMF power spectrum to high precision. Using this formulation, we summarize calculations of the effects of a PMF which take accurate quantitative account of the time evolution of the cutoff scale. We review the constructed numerical program, which is without approximation, and an improvement over the approach used in a number of previous works for studying the effect of the PMF on the cosmological perturbations. We demonstrate how the PMF is an important cosmological physical process on small scales. We also summarize the current constraints on the PMF amplitude $B_{\lambda}$ and the power spectral index $n_{\mathrm{B}}$ which have been deduced from the available $\mathrm{CMB}$ observational data by using our computational framework.
\end{abstract}

\section{Introduction}

Many astrophysical and cosmological phenomena in the universe are affected by magnetic fields over a broad range of scales. Indeed, magnetic fields with a strength of $B \sim$ $1.0 \mu \mathrm{G}$ have been detected [1-3] even on scales as large as that of galactic clusters. Such magnetic fields are frozen-in to the ionized baryons. The magnetic energy density scales as $B^{2} \propto a^{-4}$ while the baryon density scales as $\rho \propto a^{-3}$. Hence, we can relate the field strength to the cosmic density $B^{3} \propto \rho^{2}$. Considering that clusters of galaxies observed today would have isotropically collapsed relative to the background density, a cluster magnetic field of $B \sim 1.0 \mu G$ would correspond to a primordial magnetic field (PMF) of order $\sim 1.0 \mathrm{nG}$ at the epoch of photon last scattering, $z \sim 1100$.
The origin of such a cosmological primordial magnetic field has been an area of active research by many authors. One expects that such a field would have a random distribution of orientations and field strength. If the PMF has a nearly scale invariant spectrum, an origin from vector potentials generated during the inflation epoch is one of the best candidates [4-6]. Cosmological phase transitions could also produce a PMF with a bluer spectrum [7-10]. Several authors have also discussed magnetic field generation on smaller scales during or after the epoch of photon last scattering $(z \lesssim 1100)$ [11-13]. Since each model for the generation of the PMF involves different length scales, the spectral index of the PMF power spectrum, $n_{\mathrm{B}}$, correlates with the generation models. Therefore, constraining $n_{\mathrm{B}}$ can lead to constraints on models for the generation of the PMF. 
A primordial magnetic field (PMF) affects electrons and protons through the Lorentz force. Subsequently, ionized baryons affect photons through Thomson scattering. In this way, the PMF affects photons indirectly in the early universe. A number of studies in the literature [14-40] have analyzed effects of a fiducial PMF of order $\sim 1 \mathrm{nG}$ on the matter power spectrum as well as the temperature fluctuations and polarization anisotropies of the cosmic microwave background (CMB). Many others have attempted to constrain the strength of the PMF by various means, for example, the non-Gaussianity of the temperature fluctuations of CMB [41-43], Faraday rotation effects [44-47], CMB anomalies $[36,48,49]$, and effects on large-scale structure (LSS) [50, 51]. These studies indicated that the PMF effects are mainly manifest on the smallest scales in the linear regime, that is, before the formation of nonlinear structure. This is of interest because there are possible discrepancies between observation and theory in the linear regime on small angular scales which could be attributable to a PMF. However, to accurately estimate the influence of not only a PMF but also nonlinear effects, it is crucial to have the proper tools to constrain the PMF from cosmological observations. The purpose of this paper is to summarize the development of such tools.

We have developed methods [25-29, 32] to analyze the effects of a PMF on the matter and CMB power spectra. The parameters characterizing the PMF have then been constrained from a fit [38] to the observational data. By simultaneously fitting the matter and $\mathrm{CMB}$ contributions, we have shown that a more comprehensive and accurate model for the PMF effects in cosmology can be obtained. This allows for improved constraints on the parameters characterizing the PMF.

In Section 2 of this paper, we will introduce the basic cosmological physics of the PMF; and in Sections 3 and 4, we will discuss its effects on the matter power spectrum and CMB. In Section 5, we will discuss the results of a search for constraints on parameters of the PMF from observations of the matter power spectra and CMB. In Section 4, we will also explore the possibilities for new physics to be obtained from a better understanding of the PMF, for example, contributions of the PMF to the gravitational wave mode (BB-mode).

\section{The Model}

In this section, we review how to model the effects of a PMF on the cosmic fluid. We assume a flat FriedmannRobertson-Walker (FRW) background cosmology for the linear perturbations and adopt a conformal synchronous gauge as in [25]. Hence, the line element is given [25] by

$$
d s^{2}=a^{2}(\tau)\left[-d \tau^{2}+\left(\delta_{i j}+h_{i j}\right) d x^{i} d x^{j}\right],
$$

where the $x^{i}$ are spatial coordinates, $a(\tau)$ is the scale factor, $h_{i j}$ is the metric perturbation around the background spacetime, and $\tau$ is the conformal time defined by

$$
\tau \equiv \int_{0}^{t} \frac{d t^{\prime}}{a\left(t^{\prime}\right)} .
$$

Here and in the following, we use natural units $c=\hbar=1$ except where otherwise noted.

2.1. Primordial Magnetic Field. In order to solve the perturbed Einstein Equations, it is first necessary to specify the energy momentum tensor for the magnetic field. We can adopt a prior upper limit on the PMF amplitude of order $\simeq 1.0-10 \mathrm{nG}$ based upon observations of magnetic fields on the scale of galactic clusters as described below. Fortunately, for a PMF of order 1.0-10 nG at the surface of photon last scattering, the total energy density in the PMF is smaller than the energy density in the temperature fluctuations of the CMB. Therefore, we can treat the energy density of the PMF as a first-order perturbation and assume a stiff source for the time evolution. By a stiff source, we mean that all back reactions from the fluid onto the magnetic field can be discarded because these are second-order perturbations. In this case, we can also assume that the conductivity of the primordial plasma is very large and that the electric field is negligible, that is, $E \sim 0$. This "frozen-in" condition is a very good and useful approximation [15].

On the largest scales, the time evolution of the PMF can be decoupled from its spatial dependence, that is, $\mathbf{B}(\tau, \mathbf{x})=\mathbf{B}_{\mathbf{0}}(\mathbf{x}) / a^{2}$. This leads to the following simplified electromagnetic energy-momentum tensor,

$$
\begin{aligned}
& T_{[\mathrm{EM}]}^{00}(\mathbf{x}, \tau)=\frac{B(\mathbf{x})^{2}}{8 \pi a^{6}}, \\
& T_{[\mathrm{EM}]}^{i 0}(\mathbf{x}, \tau)=T_{[\mathrm{EM}]}^{0 k}(\mathbf{x}, \tau)=0, \\
& -T_{[\mathrm{EM}]}^{i k}(\mathbf{x}, \tau)=\sigma_{\mathrm{B}}^{i k}=\frac{1}{8 \pi a^{6}}\left\{2 B^{i}(\mathbf{x}) B^{k}(\mathbf{x})-\delta^{i k} B(\mathbf{x})^{2}\right\} .
\end{aligned}
$$

When dealing with cosmological fluctuations, it is convenient to work in $k$-space and denote all quantities by their Fourier transform $F(\mathbf{k})=\int d^{3} x \exp (i \mathbf{k} \cdot \mathbf{x}) F(\mathbf{x})$, where $k$ is a wave number. Just as for the CMB temperature fluctuations, a PMF that is statistically homogeneous, isotropic, and random, the fluctuation power spectrum can be parameterized as a power-law $P(k) \propto k^{n_{\mathrm{B}}}[15,24]$, where $n_{\mathrm{B}}$ is the spectral index.

The electromagnetic stress-energy tensor in $k$ space is given by [25]

$$
\begin{aligned}
& T_{j}^{i}(\mathbf{k})_{[\mathrm{EM}]} \\
& =\frac{1}{4 \pi a^{4}} \int \frac{d^{3} k^{\prime}}{(2 \pi)^{3}}\left\{\frac{1}{2} \delta_{j}^{i} B^{l}\left(\mathbf{k}^{\prime}\right) B_{l}\left(\mathbf{k}-\mathbf{k}^{\prime}\right)-B^{i}\left(\mathbf{k}^{\prime}\right) B_{j}\left(\mathbf{k}-\mathbf{k}^{\prime}\right)\right\} .
\end{aligned}
$$

When comparing with observations, one desires a statistical measure of the fluctuations on various scales. For this, it is best to work with a two-point correlation function for the PMF which then is defined [15] by

$$
\begin{array}{r}
\left\langle B^{i}(\mathbf{k}) B^{j^{*}}\left(\mathbf{k}^{\prime}\right)\right\rangle=\frac{(2 \pi)^{n_{B}+8}}{2 k_{\lambda}^{n+3}} \frac{B_{\lambda}^{2}}{\Gamma\left(\left(n_{B}+3\right) / 2\right)} k^{n_{B}} P^{i j}(k) \delta\left(\mathbf{k}-\mathbf{k}^{\prime}\right), \\
k<k_{C},
\end{array}
$$


where $B_{\lambda}=\left|\mathbf{B}_{\lambda}\right|$ is the strength of the comoving mean magnetic field derived by smoothing over a Gaussian sphere of comoving radius $\lambda$ and $k_{\lambda}=2 \pi / \lambda$ (with $\lambda=1 \mathrm{Mpc}$ here). The tensor $P^{i j}(k)$ is defined by $P^{i j}(k)=\delta^{i j}-$ $k^{i} k^{j} / k^{2}$. The damping scale from radiative viscosity provides a natural cutoff wave number $k_{C}$ in the PMF magnetic power spectrum. It is defined in [52-54].

We have evolved the PMF source power spectrum using the numerical methods described in $[25,29]$. Using this method, we have quantitatively evaluated the time evolution of the cutoff scale and, hence, more reliably calculated the effects of a PMF.

2.2. Cosmological Fluids, Curvature Fluctuations, and the $P M F$. Cosmological perturbations can be either of a scalar form (fluctuations in energy density), vector form (momentum fluctuations), or tensor form ( $T^{i j}$ fluctuations). Considering the scalar mode first, the ionized baryons (electrons and protons) in the early universe are influenced by PMF Lorentz forces. Before the epoch of photon last scattering $(z>1100)$, the photons are immersed in a fluid of ionized baryons along with the PMF and are indirectly affected by the PMF through Thomson scattering. At the same time, the matter and radiation respond to the background curvature fluctuations $\eta$. We have found that it is very important to carefully account for the dependence of the curvature fluctuations on the conformal time $\tau$ up to at least second order, $\mathcal{O}\left(\tau^{2}\right)$. This is because there is a cancellation between contributions from the primordial magnetic field and primordial radiation at first order. Fortunately, higher than first-order $\mathcal{O}(\tau)$ terms of $\eta(\tau)$ can be included by considering the matter contributions to the scale factor $a$ $[55,56]$,

$$
a \simeq \sqrt{\frac{8 \pi G \rho_{R 0}}{3}} \tau+\frac{8 \pi G \rho_{M 0}}{12} \tau^{2}=\alpha \tau+\beta \tau^{2},
$$

where $\rho_{R 0}$ and $\rho_{M 0}$ are total energy densities in radiation and matter, respectively. A model without this matter contributions may give some values which are mathematically close to the correct answer for a limited range of time and length scales. However, such models are not physically correct. In fact, by neglecting this coupling, the curvature perturbation $\eta$ of the scalar mode of our previous numerical estimation [29] was too small to stabilize the numerical calculation for large scales and early times. This problem is illustrated in Figure 1 which shows a comparison (for $B_{\lambda}=1.0 \mathrm{nG}$ and $\left.n_{\mathrm{B}}=-2.9\right)$ of the CMB temperature fluctuations in the scalar mode with and without a consideration of the matter contributions to the scale factor. Since the previous curvature perturbation $\eta$ was too small and there was an instability in the numerical calculations for large scales and early times, the CMB temperature fluctuations for lower $\ell$ rise higher without the matter contributions to $a$ than a calculation with matter contributions. In order to solve this problem, we have adopted the model of [56] for estimating the effects of the PMF on fluctuations of the scalar mode in the early universe. We have also utilized adiabatic initial conditions for the matter contributions as in [56]. This leads to stable

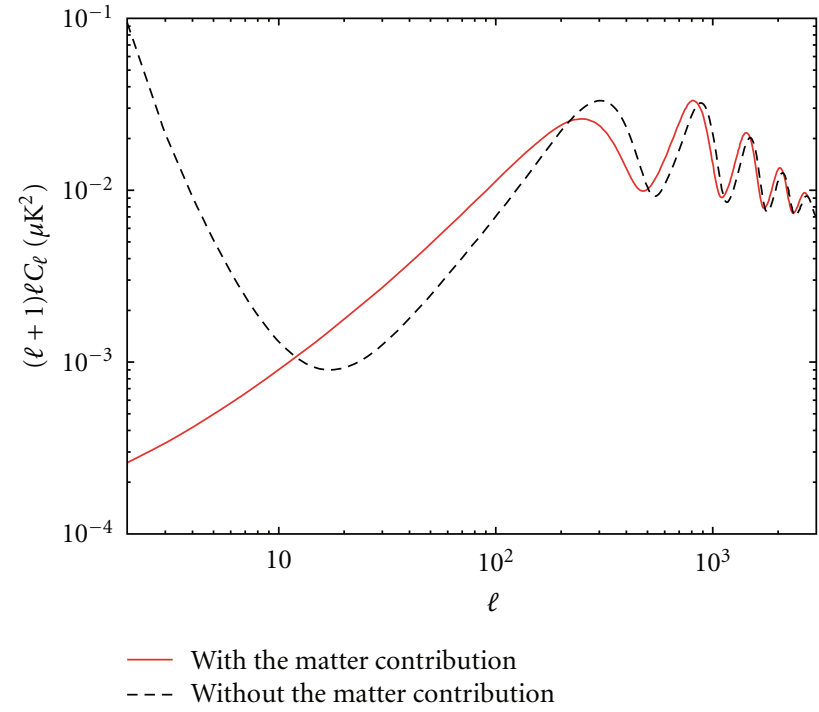

Figure 1: Comparison of CMB temperature fluctuations from the scalar mode with (red thick curve) and without (thin dashed curve) terms of $\mathcal{O}\left(\tau^{2}\right)$ from the matter contributions to the scale factor. For this plot, we have set $B_{\lambda}=1.0 \mathrm{nG}$ and $n_{\mathrm{B}}=-2.9$.

numerical calculations of the curvature perturbations of the scalar mode for all length scales and times. This is an improvement over previous numerical estimates for which the scalar curvature perturbations were too small to be stabilized in the numerical calculations for large scales and early times. Thus, our current method allows one to obtain consistent results for all scales and times of interest.

2.3. Correlations between the PMF and the Primary Density Field. Many authors have studied models for the origin of the PMF. However, there is little consensus yet as to the true origin of the PMF. Because of this, we cannot be certain of how the PMF correlates with fluctuations of the primordial density field. Nevertheless, one can define a parameter " $s$ " to denote how much the power spectrum of the PMF correlates with fluctuations of the primordial density field $[25,28]$.

In the linear regime, the power spectra of density fluctuations in the baryons $\left(P_{\mathrm{b}}(k)\right)$ and cold dark matter $\left(P_{\mathrm{CDM}}(k)\right)$ in the presence of a PMF are as follows:

$$
\begin{aligned}
P_{\mathrm{b}}(k)= & \left\langle\delta_{[\mathrm{b}: \mathrm{FL}]}(k) \delta_{[\mathrm{b}: \mathrm{FL}]}^{*}(k)\right\rangle+\left\langle\delta_{[\mathrm{b}: \mathrm{PMF}]}(k) \delta_{[\mathrm{b}: \mathrm{PMF}]}^{*}(k)\right\rangle \\
& +2\left\langle\delta_{[\mathrm{b}: \mathrm{FL}]}(k) \delta_{[\mathrm{b}: \mathrm{PMF}]}^{*}(k)\right\rangle, \\
P_{\mathrm{CDM}}(k)= & \left\langle\delta_{[\mathrm{CDM}: \mathrm{FL}]}(k) \delta_{[\mathrm{CDM}: \mathrm{FL}]}^{*}(k)\right\rangle \\
& +\left\langle\delta_{[\mathrm{CDM}: \mathrm{PMF}]}(k) \delta_{[\mathrm{CDM}: \mathrm{PMF}]}^{*}(k)\right\rangle \\
& +2\left\langle\delta_{[\mathrm{CDM}: \mathrm{FL}]}(k) \delta_{[\mathrm{CDM}: \mathrm{PMF}]}^{*}(k)\right\rangle,
\end{aligned}
$$

where the brackets denote the various two-point correlation functions as defined above, and $\delta_{\alpha}$ and $\alpha \in([\mathrm{b}: \mathrm{FL}],[\mathrm{CDM}$ : $\mathrm{FL}])$ designate the baryon and CDM density fluctuations 
without the PMF, respectively, while $\delta_{\beta}$ and $\beta \in$ ([b : $\mathrm{PMF}],[\mathrm{CDM}: \mathrm{PMF}]$ ) denote the baryon and CDM density fluctuations with the PMF included (without the primary fluctuations). In this power spectrum, we normalize the cross-correlation terms with the parameter $s$,

$$
\begin{aligned}
& \left\langle\delta_{[\mathrm{b}: \mathrm{FL}]}(k) \delta_{[\mathrm{b}: \mathrm{PMF}]}^{*}(k)\right\rangle \\
& \equiv s \sqrt{\left\langle\delta_{[\mathrm{b}: \mathrm{FL}]}(k) \delta_{[\mathrm{b}: \mathrm{FL}]}^{*}(k)\right\rangle\left\langle\delta_{[\mathrm{b}: \mathrm{PMF}]}(k) \delta_{[\mathrm{b}: \mathrm{PMF}]}^{*}(k)\right\rangle}, \\
& \left\langle\delta_{[\mathrm{CDM}: \mathrm{FL}]}(k) \delta_{[\mathrm{CDM}: \mathrm{PMF}]}^{*}(k)\right\rangle \\
& \equiv s \sqrt{\left\langle\delta_{[\mathrm{CDM}: \mathrm{FL}]}(k) \delta_{[\mathrm{CDM}: \mathrm{FL}]}^{*}(k)\right\rangle\left\langle\delta_{[\mathrm{CDM}: \mathrm{PMF}]}(k) \delta_{[\mathrm{CDM}: \mathrm{PMF}]}^{*}(k)\right\rangle} .
\end{aligned}
$$

When $0<s \leq 1, s=0$, or $-1 \leq s<0$ in (8), one has positive, vanishing, or negative correlations, respectively.

Next, the Boltzmann equation can be utilized to find the equations of the baryons as follows:

$$
\begin{aligned}
\dot{v}_{b}= & -\frac{\dot{a}}{a} v_{b}+c_{s}^{2} k^{2} \delta_{b}+\frac{4 \bar{\rho}_{\gamma}}{3 \bar{\rho}_{b}} a n_{e} \sigma_{T}\left(v_{\gamma}-v_{b}\right) \\
& +k^{2} \frac{\rho_{\gamma 0}}{a \rho_{b 0}} \frac{\prod_{[\mathrm{EM}: \mathrm{S}]}(\mathbf{k})}{4 \pi \rho_{\gamma 0}},
\end{aligned}
$$

where $v_{b}$ and $v_{y}$ are the baryon and photon velocity perturbations, $\delta_{b}$ and $\delta_{\gamma}$ are the baryon and photon density perturbations, $c_{s}$ is the sound speed, $n_{e}$ is the free electron density, $\sigma_{T}$ is the Thomson scattering cross section, $\rho_{b 0}$ and $\rho_{\gamma 0}$ are baryon and photon densities, and $\Pi_{[\mathrm{EM}: \mathrm{S}]}(\mathbf{k})$ is the square root of the power spectrum function for the Lorentz force (given in [25]).

The Lorenz force term in (9) can be divided into two terms, the magnetic pressure and the tension. By comparing those terms, one can decide which of them is dominant in the Lorenz force. There is, however, no information as to whether the magnetic pressure or the tension is dominant, and whether the direction of the forces from them is the same or different. Nevertheless, such information should be taken into account when it can be determined from a model. To see how these pieces arise, we summarize the derivation of the scalar Lorentz force term as given in [25]. Analogous derivations of the vector and tensor two-point correlation functions can be found in [29]. One can first rewrite the two-point correlation function for the scalar part of the electromagnetic energy tensor in $k$ space as

$$
\begin{aligned}
& \left\langle T_{[\mathrm{EM}: \mathrm{S}]}(\mathbf{k}) T_{[\mathrm{EM}: \mathrm{S}]}^{*}\left(\mathbf{k}^{\prime}\right)\right\rangle=\hat{k}_{i} \hat{k}^{j} \widehat{k}_{l} \hat{k}^{m}\left\langle T_{j_{[\mathrm{EM}]}^{i}}(\mathbf{k}) T_{m[\mathrm{EM}]}^{* l}\left(\mathbf{k}^{\prime}\right)\right\rangle \\
& =(2 \pi)^{3}\left|\Pi_{[\mathrm{EM}: \mathrm{S}]}(\mathbf{k})\right|^{2} \delta\left(\mathbf{k}-\mathbf{k}^{\prime}\right),
\end{aligned}
$$

where $\Pi_{[\mathrm{EM}: \mathrm{S}]}(\mathbf{k})$ is the power spectrum of the Lorenz force that we wish to analyze.

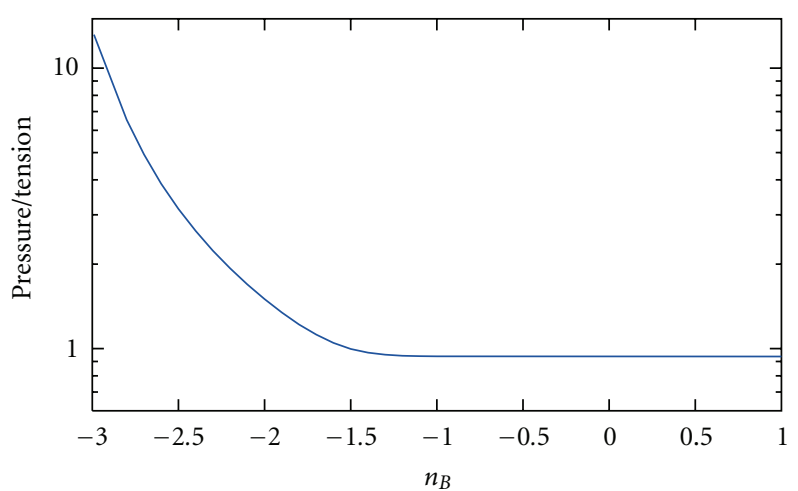

FIgURe 2: Ratio of stocahstic PMF pressure and tension sources. For illustration, the cutoff scale $k_{C}$ was fixed to $k_{C}=10 \mathrm{Mpc}^{-1}$. The pressure dominates for $n_{\mathrm{B}}<-1.5$, and the magnetic tension dominates for $n_{\mathrm{B}}>-1.5$.

One can next decompose the electromagnetic stressenergy tensor in $k$ space into two parts as follows:

$$
\begin{aligned}
T_{j}^{i}(\mathbf{k})_{[\mathrm{EM}]} & =T_{j}^{i}(\mathbf{k})_{[\mathrm{EM}: 1]}-T_{j}^{i}(\mathbf{k})_{[\mathrm{EM}: 2]}, \\
T_{j}^{i}(\mathbf{k})_{[\mathrm{EM}: 1]} & =\frac{1}{4 \pi a^{4}} \int \frac{d^{3} k^{\prime}}{(2 \pi)^{3}} \frac{1}{2} \delta_{j}^{i} B^{l}\left(\mathbf{k}^{\prime}\right) B_{l}\left(\mathbf{k}-\mathbf{k}^{\prime}\right), \\
T_{j}^{i}(\mathbf{k})_{[\mathrm{EM}: 2]} & =\frac{1}{4 \pi a^{4}} \int \frac{d^{3} k^{\prime}}{(2 \pi)^{3}} B^{i}\left(\mathbf{k}^{\prime}\right) B_{j}\left(\mathbf{k}-\mathbf{k}^{\prime}\right) .
\end{aligned}
$$

Correspondingly, one can define [25] $T_{\text {[EM:S1] }}=\hat{k}_{i} \hat{k}^{j} T_{j \text { [EM:1] }}^{i}$ and $T_{[\mathrm{EM}: \mathrm{S} 2]}=\hat{k}_{i} \hat{k}^{j} T_{j}^{i}{ }_{\text {[EM:2] }}$. Using (11), one can finally rewrite the two-point correlation function for the scalar part of the Lorenz force as

$$
\begin{aligned}
\left\langle T(\mathbf{k})_{[\mathrm{EM}: \mathrm{S}]} T^{*}\left(\mathbf{k}^{\prime}\right)_{[\mathrm{EM}: \mathrm{S}]}\right\rangle \\
=\left\langle\left(T(\mathbf{k})_{[\mathrm{EM}: \mathrm{S} 1]}-T(\mathbf{k})_{[\mathrm{EM}: \mathrm{S} 2]}\right)\right. \\
\left.\quad \times\left(T^{*}\left(\mathbf{k}^{\prime}\right)_{[\mathrm{EM}: \mathrm{S} 1]}-T^{*}\left(\mathbf{k}^{\prime}\right)_{[\mathrm{EM}: \mathrm{S} 2]}\right)\right\rangle \\
=\left\langle T(\mathbf{k})_{[\mathrm{EM}: \mathrm{S} 1]} T^{*}\left(\mathbf{k}^{\prime}\right)_{[\mathrm{EM}: \mathrm{S} 1]}\right\rangle \\
-\left\langle T(\mathbf{k})_{[\mathrm{EM}: \mathrm{S} 1]} T^{*}\left(\mathbf{k}^{\prime}\right)_{[\mathrm{EM}: \mathrm{S} 2]}\right\rangle \\
-\left\langle T(\mathbf{k})_{[\mathrm{EM}: \mathrm{S} 2]} T^{*}\left(\mathbf{k}^{\prime}\right)_{[\mathrm{EM}: \mathrm{S} 1]}\right\rangle \\
+\left\langle T(\mathbf{k})_{[\mathrm{EM}: \mathrm{S} 2]} T^{*}\left(\mathbf{k}^{\prime}\right)_{[\mathrm{EM}: \mathrm{S} 2]}\right\rangle .
\end{aligned}
$$

The first term on the R.H.S. can be identified as the magnetic pressure while the fourth term is the magnetic tension. The second and third terms are the cross correlation. A key part of the analysis of the effects of the PMF on the cosmic fluid is to understand the relative roles of these terms.

Figure 2 shows the ratio of the magnetic pressure to the tension from the Lorentz force term [25] as a function of the spectral index $n_{\mathrm{B}}$. The pressure dominates when $n_{\mathrm{B}}<-1.5$, while the tension is slightly larger when $n_{\mathrm{B}}>-1.5$. 


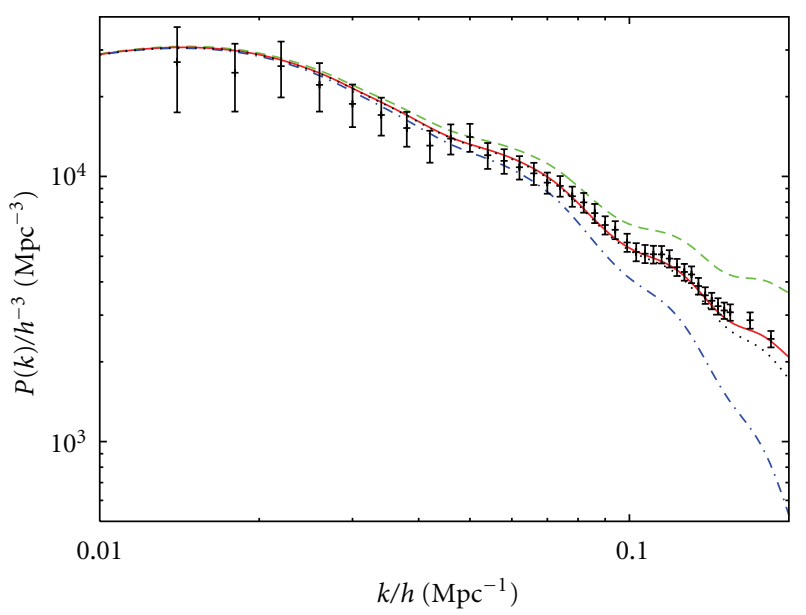

(a) $n_{\mathrm{B}}=-2.0, B_{\lambda}=3.0 \mathrm{nG}$

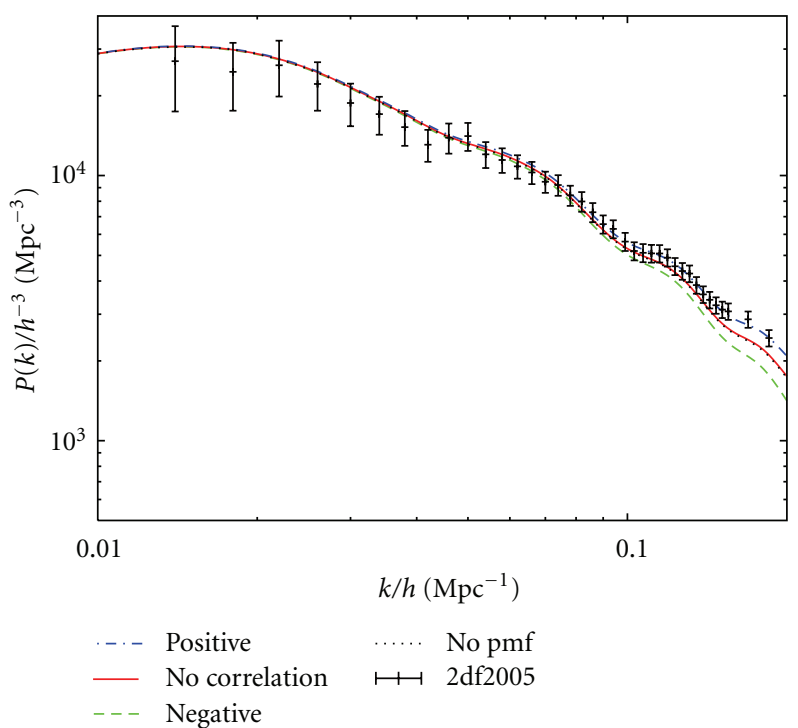

(b) $n_{\mathrm{B}}=-1.0, B_{\lambda}=3.0 \mathrm{nG}$

Figure 3: Effects on the matter power spectra of the PMF. (a) and (b) are for $n_{\mathrm{B}}=-2.0$ and -1.0 . The green-dashed, red-bold, and bluedotted-dashed curves are the case of negative $\left(s_{[\mathrm{DF}]}=-1\right)$, no $\left(s_{[\mathrm{DF}]}=0\right)$, and positive $\left(s_{[\mathrm{DF}]}=1\right)$ correlations between the matter and PMF, respectively. The black-dotted is the matter power spectrum without a PMF, and the dots with error bars show the 2df results [68].

The signs of the cross correlation terms depend upon the relative signs of the pressure and tension. In order to determine the relative signs of these two terms, however, one must specify a model for the generation of the PMF. There is as of yet no consensus model. Therefore, we decompose the factors into various possible combinations, that is,

$$
s=s_{[\mathrm{LF}]} \times s_{[\mathrm{DF}]},
$$

where

$$
\begin{gathered}
s_{[\mathrm{LF}]}=\left\{\begin{array}{lll}
-1, & n<-1.5 \quad(\mathrm{I}), \\
-1, & n>-1.5 \quad(\mathrm{II}), \\
1, & n>-1.5 \quad(\mathrm{III})
\end{array}\right. \\
0<s_{[\mathrm{DF}]} \leq 1 \quad \text { (i), } \\
s_{[\mathrm{DF}]}=0 \quad \text { (ii), } \\
-1 \leq s_{[\mathrm{DF}]}<0 \quad \text { (iii). }
\end{gathered}
$$

In the different regimes, $s_{[\mathrm{LF}]}$ represents either (I) the pressure-dominated case; (II) the tension-dominated case, where the magnetic field pressure and tension forces act in the same direction; (III) the tension-dominated case, where the magnetic field pressure and tension forces act in the opposite direction. On the other hand, $s_{[\mathrm{DF}]}$ represents either (i) a positive correlation between the matter and PMF distributions; (ii) no correlation; (iii) a negative correlation. Thus, if $s<0$, the matter and PMF distributions could be correlated positively $\left(s_{[\mathrm{DF}]}>0\right)$ and the PMF pressure dominates in the Lorentz term (for $n<-1.5$ ). Another possibility is that the matter and PMF distributions negatively correlate $\left(s_{[\mathrm{DF}]}<0\right)$ and the PMF tension dominates in the Lorentz term (for $n>-1.5$ ) and the tension acts on the density field in the same direction as the magnetic field pressure. Yet another possibility is that the PMF tension dominates in the Lorentz term $(n>-1.5)$, but the tension acts on the density field in the opposite direction from the pressure force. In these cases, the PMF effects act like a gas pressure to oppose the gravitational collapse and therefore causes the density perturbations to more slowly evolve.

On the other hand, if $s>0$, the matter and PMF distributions could positively correlate $\left(s_{[\mathrm{DF}]}>0\right)$ and the PMF tension dominates in the Lorentz term $(n>-1.5)$ while the tension acts on the density field in the opposite direction from the pressure force. Alternatively, the matter and PMF distributions could negatively correlate $\left(s_{[\mathrm{DF}]}<0\right)$, and the PMF pressure dominates in the Lorentz term $(n<-1.5)$. In these cases, the Lorentz force from the PMF accelerates the gravitational collapse. After decoupling, $\delta$ does not oscillate, and the perturbation evolution is straightforward for all of the above cases.

\section{Effects of a PMF on the Matter Power Spectrum}

In this section, we review the effects of the PMF on fluctuations in the matter density on cosmological scales (see [25] for details).

3.1. Effects of a PMF on the Matter Density Field. Figure 3 shows that the density fluctuations of matter are more strongly affected by a PMF for wavenumbers $k / h>$ $0.1 \mathrm{Mpc}^{-1}$ [25]. This is because the energy density of the PMF, $E_{\mathrm{B}}$, only depends upon the scale factor as $a^{-4}$ like the photons; and unlike the photons, the magnetic field 
fluctuations can survive on scales below the photon diffusion length, that is, below the Silk damping scale. Since the CDM and baryons interact with each other through gravity, the Lorenz force from the PMF can also indirectly affect the cold dark matter (CDM). This effect on the CDM is much smaller than that on the baryons before the epoch of photon last scattering because the density of baryons oscillates with that of photons and their gravitational effect on the CDM is very small. After the epoch of photon last scattering, the time evolution of the CDM density starts to be influenced by the baryon density through gravitational interaction [57]. Therefore, the Lorenz force effect of the PMF on the CDM increases with time.

The strength of this effect is dependent upon the ratio of the baryon density to the CDM density, $\Omega_{b} / \Omega_{c}$. The density fluctuations of the baryons are directly generated by the PMF, while the density fluctuations of the CDM are indirectly generated from the gravitational interaction with the baryons. Therefore, the density fluctuations of the CDM only grow due to gravitational instability, and their growth rate is nearly the same as the density fluctuations of the primordial CDM. On the other hand, the evolution of the baryon density fluctuations is not dominated by the gravitational potential until the gravity becomes comparable to the Lorentz force, that is, $4 \pi G \rho \delta \sim k^{2}\left(\Pi_{[\mathrm{EM}: \mathrm{S}]}(\mathbf{k}, \tau) / 4 \pi \rho_{b}\right)$, where $\Pi_{[\mathrm{EM}: \mathrm{S}]}(\mathbf{k}, \tau)$ is the Lorentz force power spectrum [25]. This is because the baryons density fluctuations are generated by the PMF directly. As mentioned above, the effect of a PMF on the baryon density fluctuations is largest on the smallest scales (larger $k$ values). Hence, the density fluctuations for baryons in the presence of a PMF increase with the wavenumber $k$ [25].

3.2. Matter Power Spectra with a PMF. It should be noted that the effect of a PMF on the matter power spectrum, $P(k)$, is different from the effect of a PMF on the matter density fluctuations $\delta$. The fluctuations of total density $\delta$ can become smaller or larger depending upon whether the dominant effect is from the pressure or the tension of the PMF. On the other hand, the effects of a PMF upon the matter power spectrum are dependent on how well the spectrum of the PMF correlates with the primary density fluctuations.

Figure 3 illustrates the degeneracy between the PMFmatter-density correlation factor $s_{[\mathrm{DF}]}$ and the PMF spectral index $n_{\mathrm{B}}$. This figure shows that a change of spectral index from $n_{\mathrm{B}}=-2.0$ to -1.0 can be offset by including a negative correlation. The correlation factor $s_{[D F]}$, however, depends upon the origin of the PMF. We will discuss below the resolution of this degeneracy problem, along with the relation between the correlation factor $s_{[D F}$ and the origin of the PMF.

3.3. Negative Correlation of the Density Perturbations. In the case of a negative correlation between the PMF and the matter density fluctuations, the pressure of the PMF accelerates the evolution of the fluctuations in the matter density, while the tension of the PMF delays them. Thus, for $n<-1.5$, for which the pressure of the PMF dominates, the PMF accelerates the evolution of the matter density fluctuations. On the other hand, for $n>-1.5$, for which the tension dominates, the PMF delays the evolution. If we assume that the PMF would have been produced in lowdensity regions, such negative correlations might be allowed. This situation, however, is difficult to realize for a causally produced PMF, because it seems natural that more amplitude for the PMF would be generated in regions with higher matter density and therefore higher currents.

3.4. No Correlation. The PMF and the matter density fluctuations are uncorrelated when $s_{[D F]}=0$. The PMF then increases the matter power spectrum independently of whether the pressure or tension dominates the PMF. If density fluctuations generate the PMF as in [13], peaks in the amplitude of the PMF should lie at peaks in the pressure gradient of the cosmological fluids. In this case, the PMF is produced along the border between high- and low-density regions, that is, $\delta \sim 0$. Eventually, such a PMF has no (or very weak) statistical correlation with fluctuations in the matter density.

3.5. Positive Correlation. In the case of a positive correlation between the PMF and fluctuations in the matter density, the evolution of the matter density fluctuations is accelerated by the tension of the PMF. Therefore, the matter evolution is delayed by the pressure of the PMF. Thus, for $n<-1.5$, for which the pressure of the PMF dominates, the evolution of the matter density fluctuations is delayed by the PMF. On the other hand, for $n>-1.5$, a PMF accelerates the evolution. If we assume that the PMF would have been produced in higher density regions, such positive correlations might be allowed.

\section{Effects of a PMF on the CMB}

In this section, we review effects of a PMF on the temperature fluctuations and polarization anisotropies of the CMB (see $[29,38]$ for details). Regarding the CMB polarization, the reader should be aware that in cosmology the intensity of polarized radiation is expressed in terms of two scalar fields $E$ and $B$ that are independent of how the coordinate system is oriented. $E$ and $B$ are the curl-free and curl-like components of the linear polarization field. Based upon these, there are three types of fluctuations: scalar, vector, and tensor. There are then four observables: the temperature, $E$-mode, $B$ mode, and the temperature cross-polarization power spectra. From these, one can generate three power spectra TT, EE, and $\mathrm{BB}$, where $T$ is the total intensity and also three cross-spectra TE, TB, and EB. However, the only nonzero spectra are TT, $\mathrm{EE}, \mathrm{BB}$, and $\mathrm{TE}$ due to parity considerations.

Figures 4-6 show that the usual (TT mode) CMB power spectra for large multipoles $\ell$ (small angular scale) are influenced most strongly by the PMF. The first reason for this is that the energy density of the PMF scales with the cosmic expansion as $a^{-4}$ just like the photons. Unlike photons, however, the magnetic fields are not much affected by photon diffusion. As a result, the temperature fluctuations and polarization anisotropies of the $\mathrm{CMB}$ continue to be 


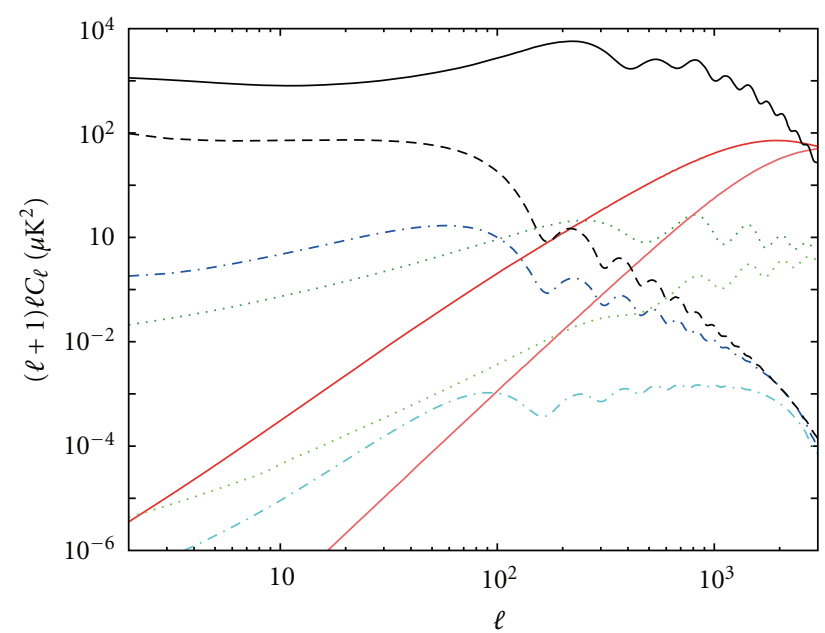

(a) TT mode

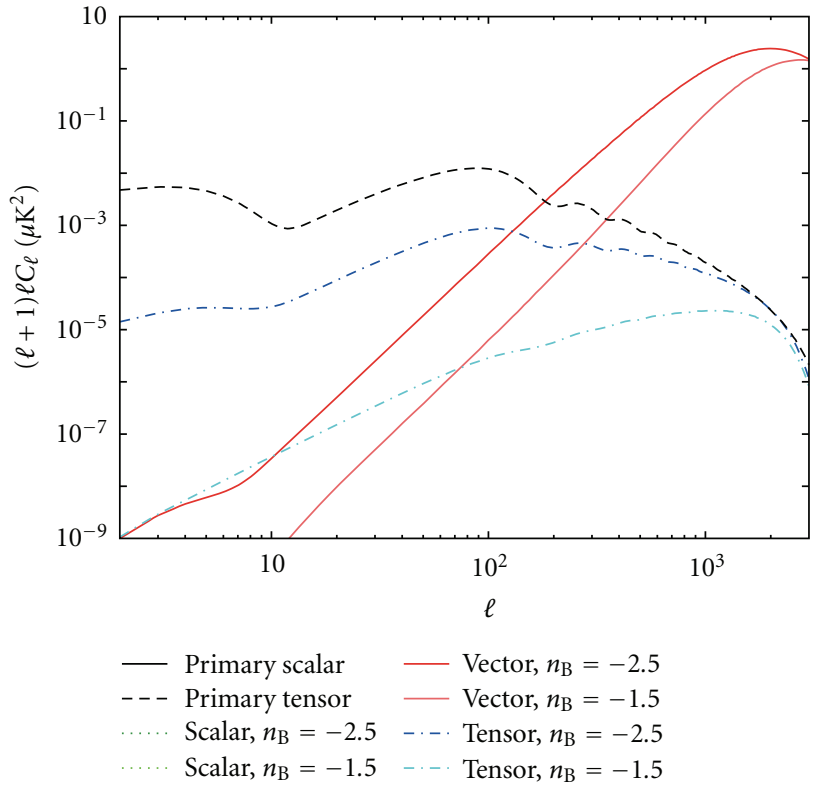

(b) BB mode

Figure 4: Polarization anisotropies and temperature fluctuations of the CMB in the from a PMF. (a) and (b) show the TT and BB modes, respectively, for models with $B_{\lambda}=3.0 \mathrm{nG}$ and $n_{\mathrm{B}}=-1.5$ or -2.5 as labeled. We use a scalar to tensor ratio of 0.2 for the primary tensor.

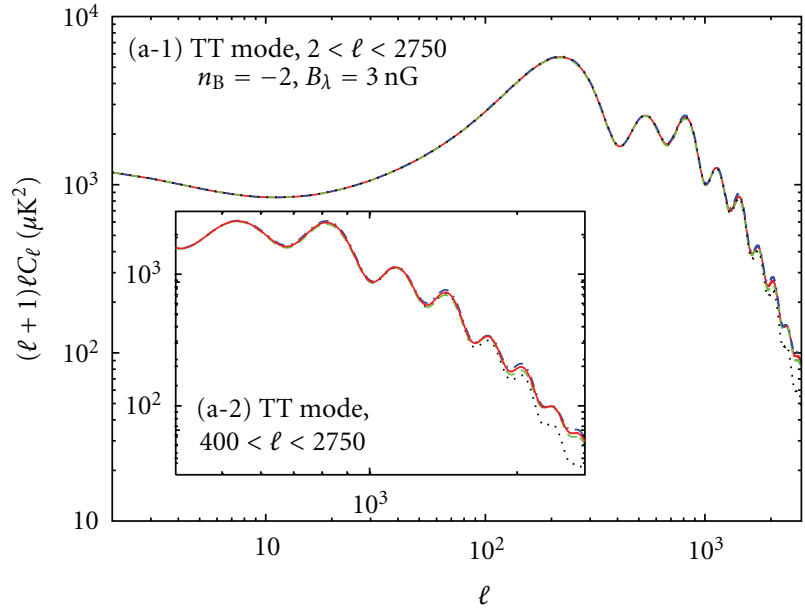

(a)

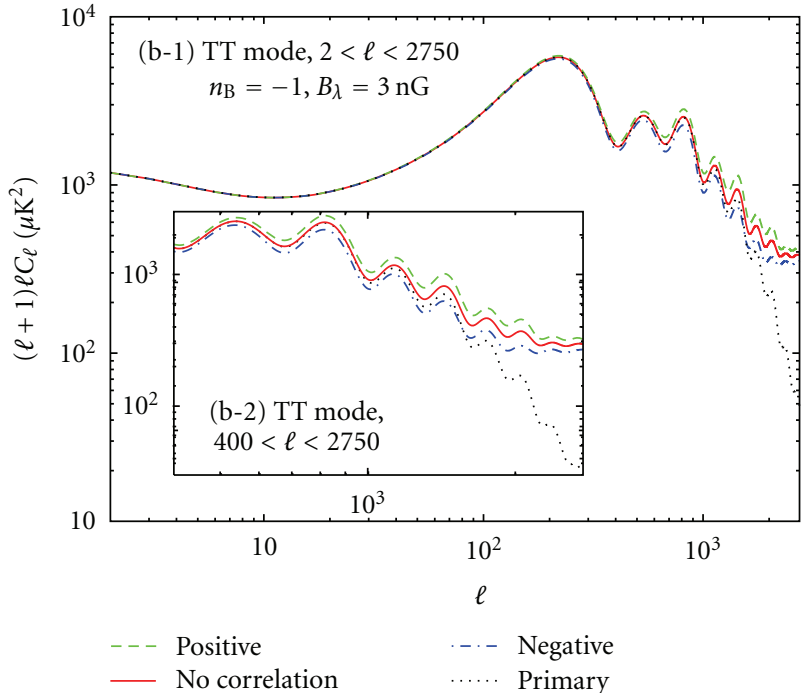

(b)

Figure 5: CMB temperature fluctuations from the PMF. Plots show various ranges for (a-1) and (b-1) TT $(2<\ell<2750)$ and (a-2) and (b-2) TT ( $400<\ell<2750)$ with $B_{\lambda}=3.0 \mathrm{nG}$ and $n_{\mathrm{B}}=-2.0$ (a) or -1.0 (b). Green-dashed, red-bold, blue-dashed-dotted, and black-dotted curves show positive, no, and negative correlations, respectively. The black-dotted curve shows the primary spectrum without a PMF.

affected by a PMF even for angular scales smaller than the Silk damping scale. Another reason is that a dominant contribution to the $\mathrm{CMB}$ on smaller angular scales comes from the vector mode of the PMF. This is because, after horizon crossing, the scalar mode cannot evolve due to the acoustic oscillations. That is, the photons fall in and out of gravitational potentials but cannot grow. On the other hand, the vector mode of the PMF fluctuations can continue growing once inside the horizon. For higher $\ell$, the vector mode also dominates the $\mathrm{CMB}$ polarization from the PMF, and the tensor mode from the PMF diminishes (cf. Figures $4-6)[17,29,38,58]$. The gravitational waves generated by the PMF can be negligibly small after horizon crossing. This is because this homogeneous solution starts to oscillate inside 


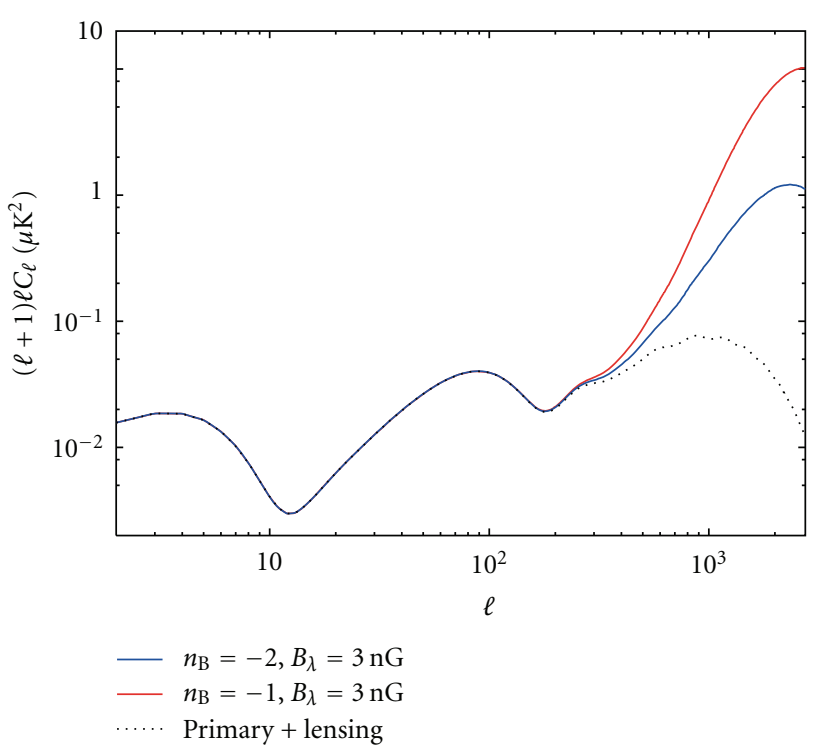

Figure 6: CMB polarization anisotropies from the PMF. Blue thin and red-bold curves show $\left(B_{\lambda}, n_{\mathrm{B}}\right)=(3.0 \mathrm{nG},-2.0)$ and $\left(B_{\lambda}, n_{\mathrm{B}}\right)=$ $(3.0 \mathrm{nG},-1.0)$. The black-dotted curve shows a primary spectrum with weak lensing effects (without the PMF).

the horizon and decay rapidly [59-62]. Consequently, the anisotropy spectrum is affected by gravity waves only on scales larger than the horizon at the epoch of photon last scattering.

Panel (b) on Figures 4 and 6 shows that the BB mode from the PMF can dominate for $B_{\lambda} \gtrsim 2.0 \mathrm{nG}$ for multipoles with $\ell \gtrsim 200$. On these angular scales, there is also the $\mathrm{BB}$ mode which is converted from the EE power spectrum by gravitational lensing [62]. Therefore, there is a degeneracy between the PMF and gravitational lensing on small angular scales. However, since the spectrum from the effects of the gravitational lensing signal has been independently determined, this power can be subtracted directly. We have shown $[29,38]$ that the effects of a PMF on the temperature fluctuations of the CMB are stronger with correlations than without, as is also the case for the matter power spectrum with a PMF. This is illustrated in Figure 5.

There is also a degeneracy between the SunyaevZel'dovich effect and the PMF. Nevertheless, the effects of a PMF on the $\mathrm{CMB}$ are independent of frequency because the PMF influences the primary $\mathrm{CMB}$ as a background. Eventually, by using observations at different frequencies, it should be possible to distinguish the effects of a PMF from foreground effects which depend upon frequency.

\section{Constraints on the PMF}

In this section, we review constraints on the parameters of the PMF from cosmological observations. First, we show that a strong constraint on the PMF amplitude and spectral index can be derived from the prior observed constraints on the $\sigma_{8}$ parameter. This fiducial quantity represents the root-mean square of the matter density fluctuation in a comoving sphere of radius $8 h^{-1} \mathrm{Mpc}$ and as such is a measure of the growth of large-scale structure. It is given by a weighted integral over the matter power spectrum [63]. Second, we note that the parameters of the PMF can be strongly constrained by using the observational data on the $\mathrm{CMB}$ with the previous priors used in the analysis of [64-66] without a PMF. In the last subsection, we will discuss the possible origins of a PMF based upon the deduced constraints on the parameters characterizing the PMF.

5.1. Prior on the PMF Parameters from $\sigma_{8}$. Because of the degeneracy of effects on the observed CMB power spectrum from various parameters, it has become standard in the field of $\mathrm{CMB}$ physics to fit the $\mathrm{CMB}$ parameters by a Baysian method that makes optimum use of the variety of independently determined constraints on cosmological parameters. For this purpose, the Monte-Carlo Markov Chain (MCMC) [67] analysis has become the method of choice to find the multidimensional surface of maximum likelihood among the many parameters. In this context, we note that the PMF amplitude $B_{\lambda}$ and power law index $n_{\mathrm{B}}$ have a strong degeneracy. Therefore, one must identify a strong prior constraint to effectively decouple these two parameters of the PMF. In this quest, we are aided by observed constraints on the $\sigma_{8}$ parameter for which a value of $0.7<\sigma_{8}<0.9$ has been deduced from diverse observational data on linear cosmological scales [68-71]. Using this range as a prior in the Monte-Carlo Markov Chain (MCMC) [67] analysis, constraints on the parameters of the PMF can be obtained by a fit to the observed power spectra of the CMB. For some of the cosmological parameters, $\Omega_{b}, \Omega_{\mathrm{CDM}}$, $n_{\mathrm{S}}$, and $A_{\mathrm{S}}$ affect $\sigma_{8}$, we also have to consider the possible degeneracy between these cosmological parameters and the PMF parameters. Fortunately, however, recent observations of the $\mathrm{CMB}$ have constrained such cosmological parameters on larger scales $(\ell<1000)$ [64-66]. As mentioned above, the PMF mainly affects the CMB anisotropies on smaller scales $(\ell>1000)[25,26,29]$. Therefore, only a small degeneracy between the PMF parameters and the other cosmological parameters is expected, so that fixing the other cosmological parameters at the WMAP best fit values is justified.

Figure 7 and the panels in Figure 8 show the optimum PMF parameters $n_{\mathrm{B}}$ and $B_{\lambda}$ for various constant values of $\sigma_{8}$ as labeled. Since the PMF power spectrum depends upon $k^{2 n_{\mathrm{B}}+3}$ [29], for $n_{\mathrm{B}}<-1.5$, the PMF effects on the density fluctuations for smaller scales decrease with lower values for $n_{\mathrm{B}}$. Therefore, since $\sigma_{8}$ depends upon the amplitude of the matter power spectrum for smaller scale, when the spectral index is near $n_{\mathrm{B}}=-3.0$, the matter power spectrum in the presence of a PMF is smaller for smaller scales. Thus, larger amplitudes of $B_{\lambda}$ are allowed. For $n_{\mathrm{B}}>-1.5$, however, the energy density power spectrum of the PMF is proportional to the cutoff scale $k_{C}^{2 n_{B}+3}$ [29]. $k_{C}$ is also proportional to $B_{\lambda}^{-1 /\left(n_{B}+5\right)}[29,52-54]$. Substituting these relations into (15)(17) in [29], we easily obtain the following relation between the PMF power spectrum and the magnetic field strength, $P(k)_{\mathrm{PMF}} \propto B_{\lambda}^{\left[14 /\left(n_{\mathrm{B}}+5\right)\right]}$. Considering $B_{\lambda} \ll 1 \mathrm{G}$, the matter power spectrum with the PMF for $n_{\mathrm{B}}>-1.5$ becomes larger 


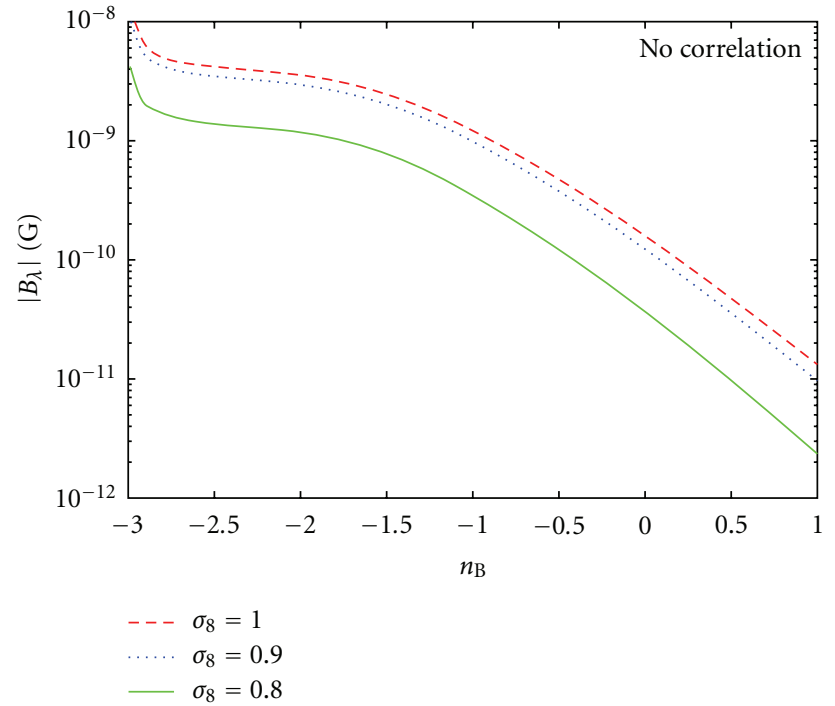

FIGURE 7: Curves of constant values for the $\sigma_{8}$ in the parameter plane of PMF amplitude $B_{\lambda}$ versus spectral index $n_{\mathrm{B}}$ for the case of no correlation between the PMF and the matter density fluctuations. Red-dashed, blue-dotted, and green-bold curves show constant values of $\sigma_{8}=1.0,0.9$, and 0.8 , respectively.

for larger $n_{\mathrm{B}}$. Therefore, larger amplitudes of $B_{\lambda}$ are not allowed for larger $n_{\mathrm{B}}$.

Since the effects of a PMF for negative and positive correlations change at $n_{\mathrm{B}}=-1.5$ (see Section 2.3), we divide the discussion below of each correlation into two parts based upon whether the spectral index is greater or less than $n_{\mathrm{B}}=$ -1.5 .

5.1.1. No Correlation. As mentioned above in Section 3.4, in the case of no correlation between the PMF and the density fluctuations from primary perturbations, the effect of a PMF is to increase the matter power spectrum independently of whether the pressure or tension dominates the PMF. As noted above, from recent constraints on the $\sigma_{8}$ parameter from cosmological observations of LSS, we can exclude PMF parameters which imply $\sigma_{8}>1$.

Hence, we can exclude a PMF with field strength $B_{\lambda} \gtrsim$ $1 \mathrm{nG}$ if $n_{\mathrm{B}}>-0.9$ (Figure 7). We can also exclude a PMF with amplitude $B_{\lambda} \gtrsim 0.1 \mathrm{nG}$ if $n_{\mathrm{B}}>0.2$. If we assume that the origin of the observed magnetic field in the clusters of galaxies is the PMF, we can limit the PMF amplitude to $B_{\lambda} \gtrsim$ $1 \mathrm{nG}$. In this case, we can exclude a PMF spectral index in the range $n_{\mathrm{B}}>-0.9$.

5.1.2. Negative Correlation. As noted above in Section 3.3, in the case of a negative correlation, the tension of the PMF delays the evolution of the matter density fluctuations, while the pressure tends to accelerate them. Furthermore, the pressure of the PMF dominates for $n_{\mathrm{B}}<-1.5$, while the tension of the PMF dominates for $n_{\mathrm{B}}>-1.5$. Therefore, for $n<-1.5$, the matter density fluctuations are accelerated by the PMF, while, for $n>-1.5$, this evolution is delayed by the
TABLE 1: PMF parameters and $\Lambda$ CDM model parameters and $68 \%$ confidence intervals $\left(A_{t} / A_{s}\right.$ is a $\left.95 \% \mathrm{CL}\right)$ from a fit to the WMAP [73] + ACBAR [74] + CBI [75] + Boomerang [76] + 2dFDR [68] data.

\begin{tabular}{lcc}
\hline \multicolumn{3}{c}{ Cosmological parameters } \\
Parameter & mean & best fit \\
\hline$\Omega_{\mathrm{b}} h^{2}$ & $0.02320 \pm 0.00059$ & 0.02295 \\
$\Omega_{\mathrm{c}} h^{2}$ & $0.1094 \pm 0.0046$ & 0.1093 \\
$\tau_{C}$ & $0.087 \pm 0.017$ & 0.082 \\
$n_{s}$ & $0.977 \pm 0.016$ & 0.970 \\
$\ln \left(10^{10} A_{s}\right)$ & $3.07 \pm 0.036$ & 3.06 \\
$A_{t} / A_{s}$ & $<0.170(68 \% \mathrm{CL}),<0.271(95 \% \mathrm{CL})$ & 0.0088 \\
$\left|B_{\lambda}\right|(\mathrm{nG})$ & $<\mathbf{2 . 1 0}(68 \% \mathrm{CL}),<\mathbf{2 . 9 8}(95 \% \mathrm{CL})$ & $\mathbf{0 . 8 5}$ \\
$n_{\mathrm{B}}$ & $<-\mathbf{1 . 1 9}(68 \% \mathrm{CL}),<-\mathbf{0 . 2 5}(95 \% \mathrm{CL})$ & $-\mathbf{2 . 3 7}$ \\
\hline$\sigma_{8}$ & $0.812_{-0.033}^{+0.028}$ & 0.794 \\
$H_{0}$ & $73.3 \pm 2.2$ & 72.8 \\
$z_{\text {reion }}$ & $10.9 \pm 1.4$ & 10.5 \\
Age $(\mathrm{Gyr})$ & $13.57 \pm 0.12$ & 13.62 \\
\hline
\end{tabular}

PMF. This behavior can be traced to the third terms in (8). Using the allowed range of PMF parameters as mentioned above, we can exclude the range of PMF amplitude to $B_{\lambda} \gtrsim$ $1 \mathrm{nG}$ if $n_{\mathrm{B}}>-0.81$, and the range of PMF amplitudes to $B_{\lambda} \gtrsim 0.1 \mathrm{nG}$ if $n_{\mathrm{B}}>0.26$ (Figures $8(\mathrm{a})$ and $8(\mathrm{~b})$ ). However, as mentioned above, it is difficult to model the production of a PMF in matter fields of low-energy density in order to realize such negative correlations.

5.1.3. Positive Correlation. As noted above in Section 3.5, in the case of a negative correlation, the pressure of the PMF delays the evolution of the matter density fluctuations while the tension accelerates them. Considering the same conditions as in the previous Section 5.1.1, the PMF decreases the fluctuations of matter density for $n_{\mathrm{B}}<-1.5$. On the other hand, for $n_{\mathrm{B}}>-1.5$, it increases the fluctuations. In this case, we can exclude the range of PMF amplitudes with $B_{\lambda} \gtrsim 1 \mathrm{nG}$ if $n_{\mathrm{B}}>-0.94$, and the range of PMF amplitudes with $B_{\lambda} \gtrsim 0.1 \mathrm{nG}$ for $n_{\mathrm{B}}>0.13$ (Figures $8(\mathrm{c})$ and $8(\mathrm{~d})$ ).

5.2. Constraint on the PMF from the CMB. In this section, we summarize the current constraints on parameters of the PMF from fits to the CMB and LSS observational data. We consider a flat $(k=0) \Lambda \mathrm{CDM}$ universe characterized by 8 parameters, that is, $\left\{\Omega_{b} h^{2}, \Omega_{c} h^{2}, \tau_{C}, n_{s}, \log \left(10^{10} A_{s}\right), A_{t} / A_{s}\right.$, $\left.\left|B_{\lambda}\right|, n_{\mathrm{B}}\right\}$, where $\Omega_{b} h^{2}$ and $\Omega_{c} h^{2}$ are the baryon and CDM densities in units of the critical density, $h$ denotes the Hubble parameter in units of $100 \mathrm{~km} \mathrm{~s}^{-1} \mathrm{Mpc}^{-1}, \tau_{C}$ is the optical depth for Compton scattering, $n_{s}$ is the spectral index of the primordial scalar fluctuations, $A_{s}$ is the scalar amplitude of the primordial scalar fluctuations, and $A_{t}$ is the scalar amplitude of the primordial tensor fluctuations. We define the tensor index for the primordial tensor fluctuations as $n_{t}=-\left(A_{s} / A_{t}\right) / 8$. For all cosmological parameters, we use the same prior constraints as those adopted in the WMAP analysis [72]. As noted in the previous section, the case of 


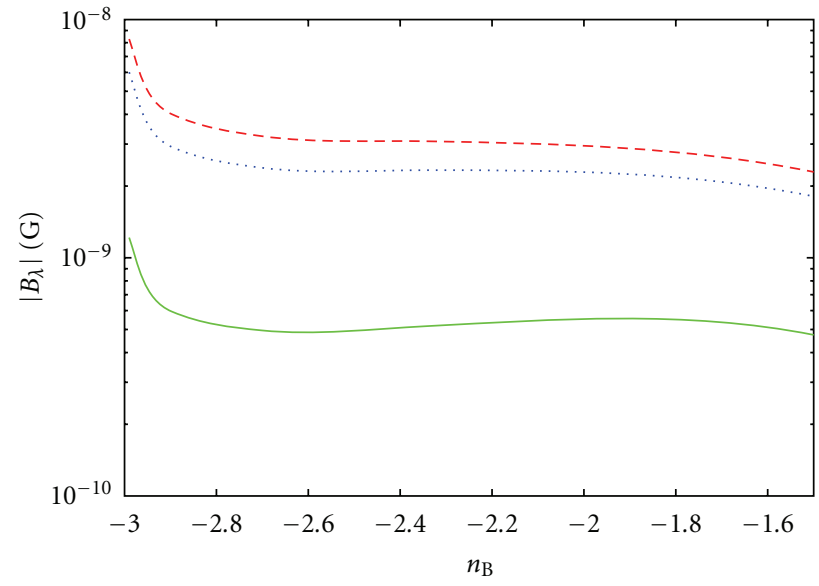

(a) Negative correlation

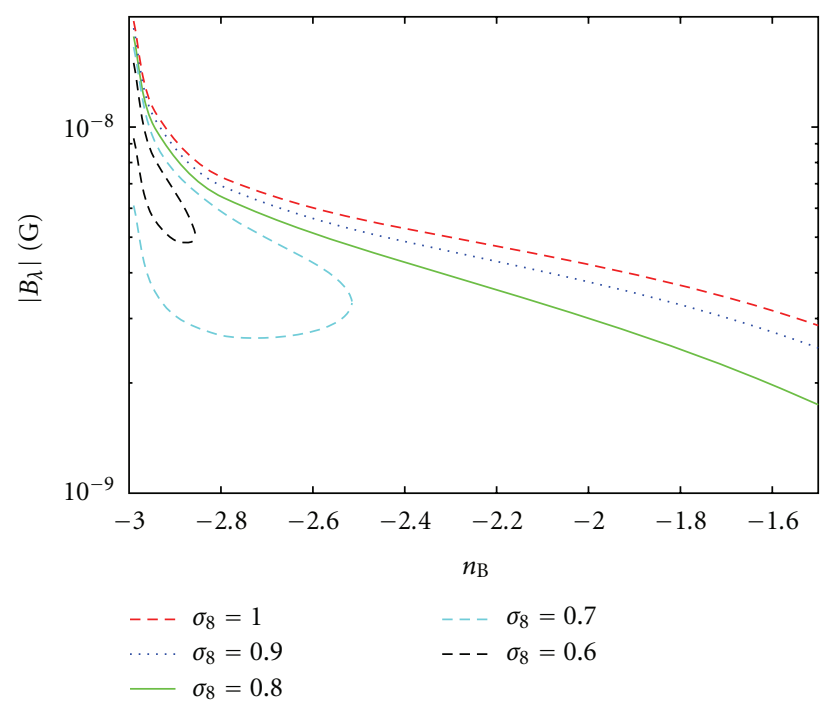

(c) Positive correlation

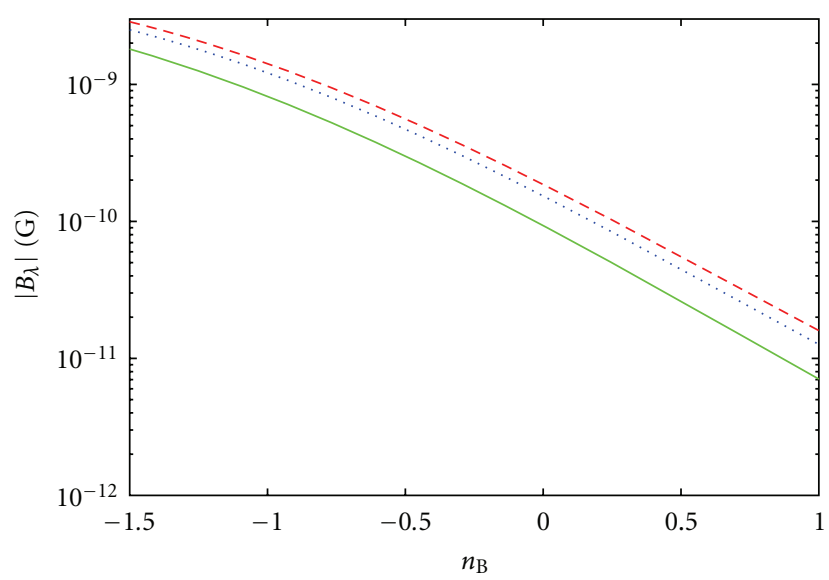

(b) Negative correlation

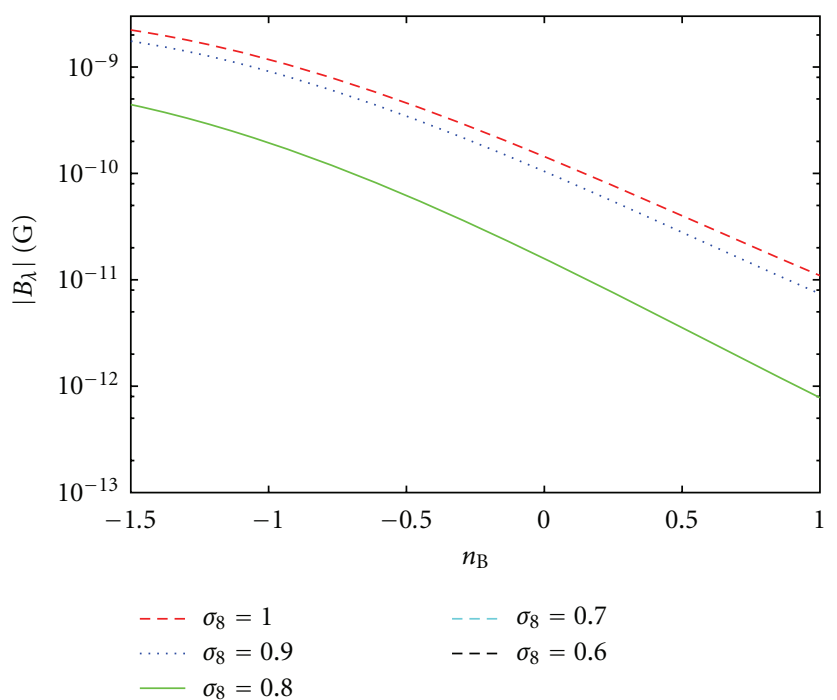

(d) Positive correlation

Figure 8: Curves of constant values for the $\sigma_{8}$ in the parameter plane of PMF amplitude $B_{\lambda}$ versus spectral index $n_{\mathrm{B}}$ for the negative $\left(s_{[\mathrm{DF}]}=-1\right)$ and positive $\left(s_{[\mathrm{DF}]}=1\right)$ correlations between the PMF and the matter density fluctuations. Panels (a) and (c) show $n_{\mathrm{B}}<-1.5$ as the PMF pressure-dominated region. On the other hand, panels (b) and (d) show $n_{\mathrm{B}}>-1.5$ as the PMF tension-dominated region. Red-dashed, blue-dotted, green-bold, azure-dotted thin, and black-dashed thin curves show constant values of $\sigma_{8}=1.0,0.9,0.8,0.7$, and 0.6 , respectively.

no correlation is more realistic than the case of negative or positive correlations. Therefore, we focus here on the constraints of the parameters characterizing the PMF in the case of no correlation.

Using the MCMC algorithm and available cosmological observations, we have constrained the standard cosmological parameters and the PMF parameters listed in Table 1. We note, however, that the MCMC algorithm tends to sparsely sample near a boundary and hence find low probability there. This is why it appears that $n_{B}$ is constrained even in the limit that $B_{\lambda} \rightarrow 0$. Of course, for $B_{\lambda}=0$, there is no constraint on the spectral index at all. This shortcoming, however, does not negate the fact that there is a genuine minimum in the goodness of fit for a finite magnetic field and spectral index. In the optimum fit with the inclusion of parameters of the PMF, the minimum total $\chi^{2}$ changes from 2803.4 to 2800.2 corresponding to a change in the $\chi^{2}$ per degree of freedom from 1.033 to 1.031 . Therefore, a finite PMF slightly improves the goodness of fit even after taking into account the new degrees of freedom. The existence of a PMF, however, is still only of marginal significance.

Figures 9 and 10 show the $68 \%$ and 95\% C.L. probability contours in the planes of various cosmological parameters versus the amplitude $\left|B_{\lambda}\right|$, or power law index $n_{\mathrm{B}}$, along with the probability distributions. Figure 10 and the bottom panels in Figure 9 show the probability distributions for $\left|B_{\lambda}\right|$ and $n_{\mathrm{B}}$. Of particular note for this review is the presence of maxima in the likelihood functions for $\left|B_{\lambda}\right|=0.85 \pm 1.25 \mathrm{nG}$ 


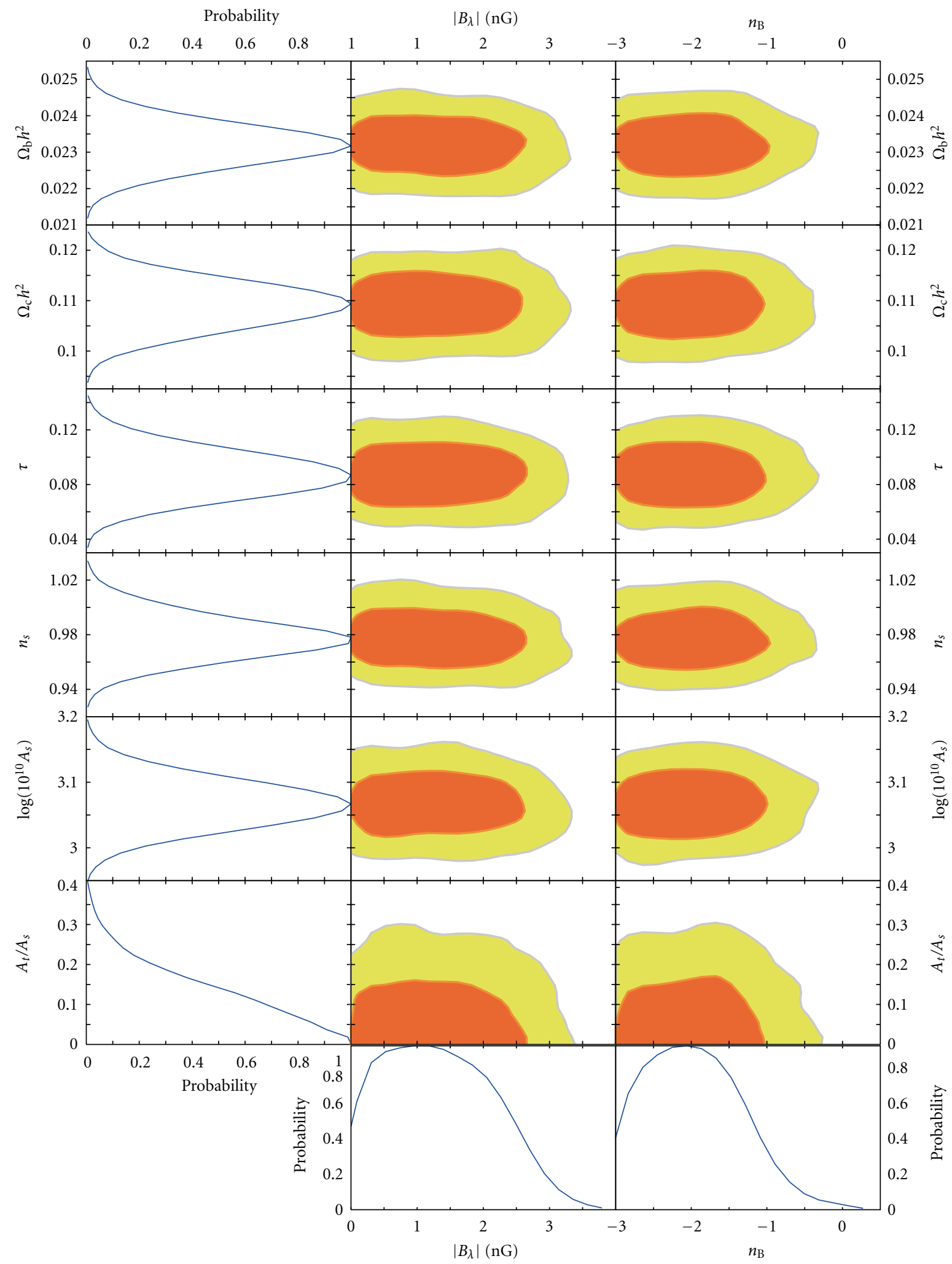

Figure 9: Probability distributions and contours of $1 \sigma$ and $2 \sigma$ confidence limits for the standard cosmological parameters as a function of the PMF field strength $\left|B_{\lambda}\right|$ and power law index $n_{\mathrm{B}}$. Orange contours show $1 \sigma(68 \%)$ confidence limits, and yellow contours show $2 \sigma(95 \%)$ confidence limits. Blue curves in the left and bottom of the figure show the probability distributions of each parameter. Note the existence of a maximum in the probability distributions for $\left|B_{\lambda}\right|$ and $n_{\mathrm{B}}$. The standard cosmological parameters do not have a degeneracy with the PMF parameters because they are mainly constrained by the observed CMB data for $\ell<1000$ (up to the 2nd peak), while the PMF is mainly influenced by the power on smaller angular scales and higher multipoles, $\ell>1000$. 


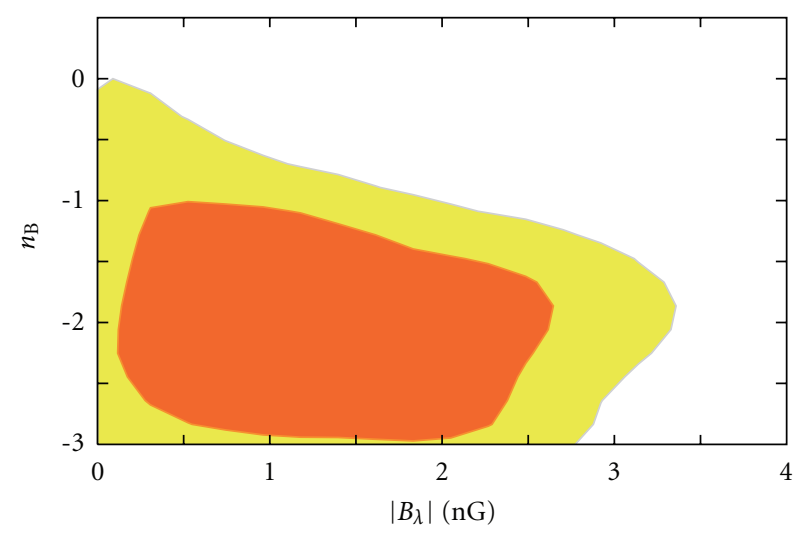

Figure 10: Probability contours in the plane of $B_{\lambda}$ versus $n_{\mathrm{B}}$. Orange contours show $1 \sigma(68 \%)$ confidence limits, and yellow contours show $2 \sigma(95 \%)$ confidence limits.

and $n_{\mathrm{B}}=-2.37_{-0.73}^{+0.88}$. Although these values are consistent with zero magnetic field, and thus they only imply upper limits, they suggest the possibility that with forthcoming data (particularly for large CMB multipoles) a finite magnetic field may soon be detectable.

These figures exhibit no degeneracy between the PMF parameters and the standard cosmological parameters. Table 1 confirms that the standard cosmological parameters are not significantly different from those deduced directly from the WMAP 5-year data analysis without a PMF. The reason for this is simple. The standard cosmological parameters are mainly constrained by the observed CMB power spectrum for low multipoles $\ell<1000$ (up to the 2 nd acoustic peak). On the other hand, the PMF dominates for $\ell>1000$. Hence, the PMF effect on the power spectrum is nearly independent of the standard cosmological parameters.

The tensor-to-scalar ratio $A_{t} / A_{s}$ deduced in our analysis is smaller than the upper limit $A_{t} / A_{s}<0.43$ (95\% CL) deduced from the WMAP 5-year data analysis without a PMF. The reason for this is that we define $A_{t}$ as the tensor amplitude of the primary $\mathrm{CMB}$ spectrum (without a PMF). This tensor term only arises from the primordial gravitational-wave background produced during inflation. We combine the tensor amplitude from the PMF and $A_{t}$ when we compare our tensor amplitude with the result deduced by others. The value of $A_{t}$ by itself is comparable to the tensor contribution from the PMF. The value of $A_{t} / A_{s}$ from the WMAP 5-year data is less than 0.43 (95\% CL). Hence, our result is consistent with the previous constraints when the additional PMF contribution is included.

The degeneracy of the PMF parameters [26] is broken by the different effects of the PMF on both the matter power spectrum and the $\mathrm{CMB}$ power spectrum. The vector mode can dominate for higher $\ell$ in the CMB temperature anisotropies [29], while the matter power spectrum becomes sensitive [25] to the power law spectral index $n_{\mathrm{B}}$ when a PMF is present. Additionally, the $\mathrm{CMB}$ fluctuations from the $\mathrm{PMF}$ are smaller than the primary CMB fluctuations for the scalar and tensor modes on large angular scales $[30,31]$. Therefore, the tensor-to-scalar ratio is not affected by a PMF.
Figure 11 shows our deduced probability distributions and the $1 \sigma$ and $2 \sigma$ (68\% and 95\% CL) probability contours for the resultant cosmological parameters, $\sigma_{8}, H_{0}, z_{\text {reion }}$, and Age. Here, $H_{0}=100 \mathrm{hm} \mathrm{s}^{-1} \mathrm{Mpc}^{-1}$ is the Hubble parameter, $z_{\text {reion }}$ is the red shift at which reionization occurs, and Age is the presently observed age of the universe in Gyr. It is important to keep in mind that these parameters are not input parameters but are output results. The sum of the results on Figures 9-11 provides marginal evidence that both upper and lower limits to the parameters of the PMF can be deduced.

Table 1 summarizes the upper limits to the PMF parameters along with the input (constrained by priors) and output (unconstrained by priors) cosmological parameters: $\sigma_{8}, H_{0}, z_{\text {reion, }}$ and Age. In particular, we find that $\left|B_{\lambda}\right|<2.10\left(68 \%\right.$ CL) nG and $<2.98$ (95\% CL) nG and $n_{\mathrm{B}}<$ $\mathbf{- 1 . 1 9}(68 \% \mathrm{CL})$ and $<-\mathbf{0 . 2 5}$ (95\% CL) at a present scale of $1 \mathrm{Mpc}$. Although previous work [25, 26, 29, 32] could obtain a less stringent upper limit to $\left|B_{\lambda}\right|$, they could not constrain $n_{\mathrm{B}}$ at all. Moreover, our deduced probability distributions suggest that a finite PMF provides the best fit.

On angular scales smaller than that probed by the CMB, the observed number density of galaxies is a better measure of the power spectrum. Therefore, since the PMF mainly influences the small angular scales, using the combined LSS observational data (2dFDR [68]) and the CMB (WMAP 5 years [73], ACBAR [74], CBI [75], and Boomerang [76]), we can constrain the PMF better than in previous works which relied on the $\mathrm{CMB}$ data only. In particular, an upper limit on $n_{\mathrm{B}}$ can be constrained for the first time while the lower limit to $n_{\mathrm{B}}$ is approaching the $1 \sigma$ confidence level.

The right-bottom panel of Figure 9 shows that the maximum likelihood is for a spectral index of $n_{\mathrm{B}}=-2.4_{-0.7}^{+0.9}$. It is important $[26,77]$ to constrain $n_{\mathrm{B}}$ as this parameter provides insight into models for the formation of the PMF. If the PMFs were formed during inflation, one would expect a scale-invariant value of $n_{\mathrm{B}}=-3$. The value deduced in Figures $8-11$ and Table 1 is thus consistent with an inflationgenerated PMF at the $1 \sigma$ confidence level.

Next, we discuss other generation models for the PMF during various phase transitions [7-10] from the gravity waves produced along with the PMF. The formation of light elements during big-bang nucleosynthesis (BBN) depends upon a balance between the nuclear reaction rates and the expansion rate of the universe. Since gravity waves contribute to the total energy density, they affect the expansion rate. Hence, they are constrained by a comparison between the $\mathrm{BBN}$ predictions and the observed light element abundances $[26,77]$. There are also other theoretical constraints on the origin of the PMF. For example, in [78], it was noted that a non-scale-invariant spectrum of the PMF is difficult to generate or survive if it is formed via a causal mechanism during later phase transitions. Indeed, as pointed out in $[77,78]$, the spectral index of a magnetic field generated during any period of standard Friedmann expansion (i.e., any time except inflation) is constrained to be a positive even integer larger than two: $n_{B}=2,4,6 \ldots$ This constraint, which applies, for example, to a PMF generated at a phase transition or by second-order MHD processes and so on, 


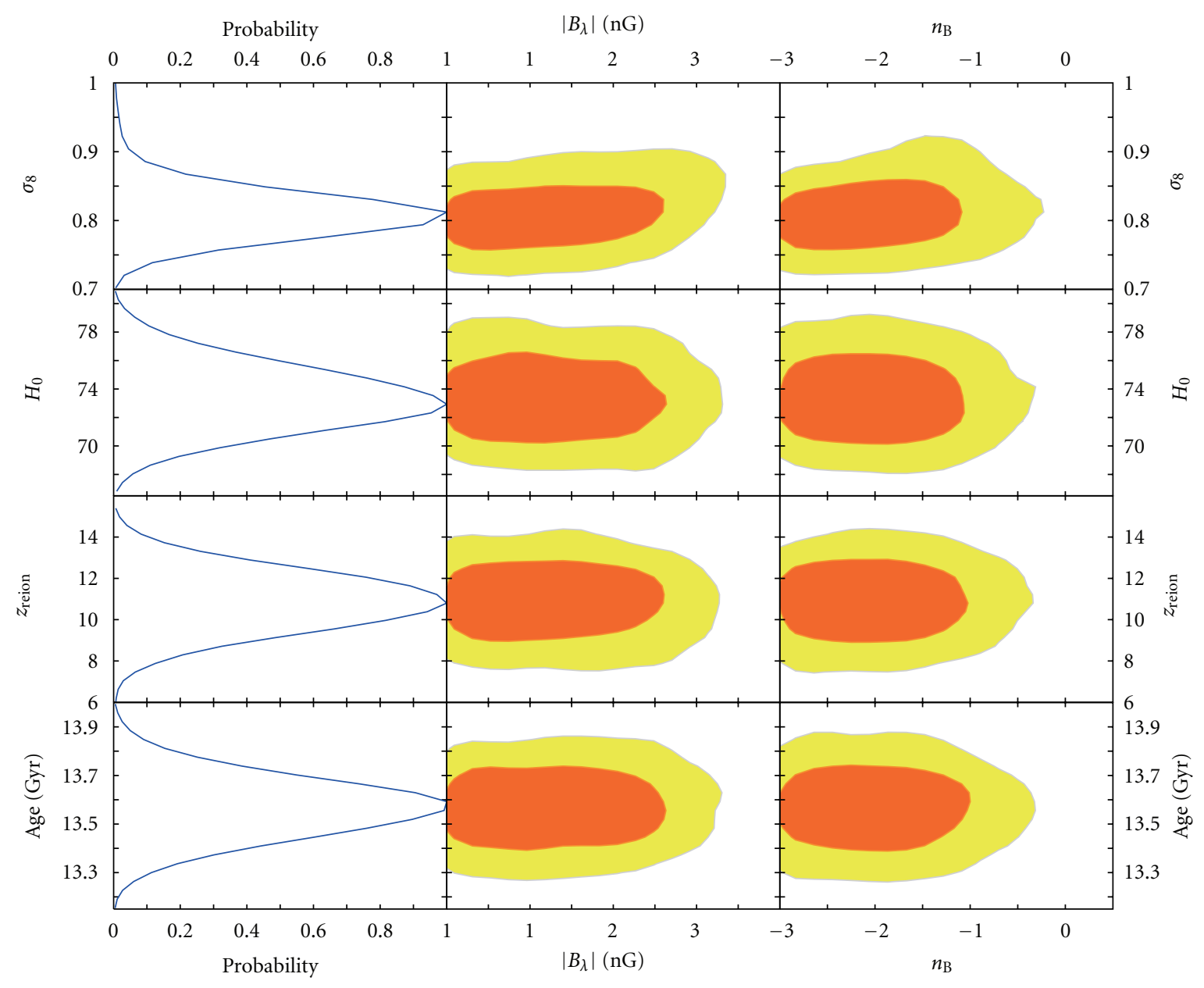

FIgURE 11: Probability distributions and contours of $1 \sigma$ and $2 \sigma$ confidence limits for the parameters $\sigma_{8}, H_{0}, z_{\text {reion }}$, and Age. Orange contours show $1 \sigma(68 \%)$ confidence limits, and yellow contours show $2 \sigma(95 \%)$ confidence limits. Blue curves show probability distributions for each parameter. Note that these are not input priors, but output results.

comes simply from the fact that the magnetic field generation is a causal process (i.e., the universe has a finite horizon at the magnetic field generation time). Based upon this, the fact our results favor a negative spectral index near -3 indicates that an inflation mechanism [4-6] may be the most likely origin for the PMF.

A PMF affects not only the temperature fluctuations, but also the polarization of the CMB. Although we fit all available polarization data, it turns out that the TT and $\mathrm{BB}$ modes (where $T$ is the temperature fluctuation and $B$ is the curl-like component of polarization) are the most important. Figures 12 and 13 show a comparison of the computed bestfit total power spectrum with the observed CMB spectrum. Plots show various spectra for the TT and BB modes. We plot the best fit and allowed regions both including the SZ effect (scattering from reionized electrons) at the $K(22.8 \mathrm{GHz})$ band (upper curves) and without the SZ effect (lower curves) in Figure 11. Including the SZ effect only slightly diminishes the best fit magnitude of the PMF. Although the CBI point falls about $1 \sigma$ above the best fit, the $\chi^{2}$ is dominated by the better ACBAR08 data, and this point does not significantly affect the deduced PMF parameters.

\section{Discussion}

Effects of a PMF on the early universe have been studied by a number of researchers including ourselves. A summary of some of the relevant topics and their implications for different PMF field strengths is given in Table 2. For example, in the first row, if a PMF originated during a postinflation epoch, its strength is probably $\left|\mathrm{B}_{\lambda}\right| \ll 1 \mathrm{nG}$, while if it was generated during inflation, its field strength at the surface of last photon scattering was likely $\left|\mathrm{B}_{\lambda}\right| \sim 1 \mathrm{nG}$. Furthermore, the $\mathrm{BB}$ mode of the $\mathrm{CMB}$ from the tensor component is strongly affected by the gravitational wave background. Therefore, the BB mode also shows characteristics of the gravitational wave background from the PMF and an inflation origin. If the PMF has a field strength order of $1 \mathrm{nG}$, then from Figures 5 and 6 , we expect that the gravity wave background (without a PMF) dominates the BB mode 


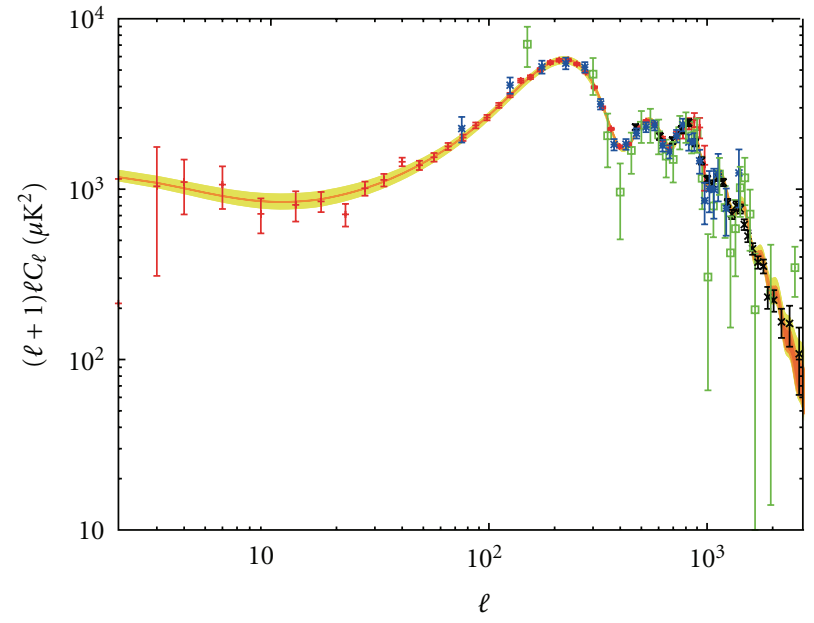

(a)

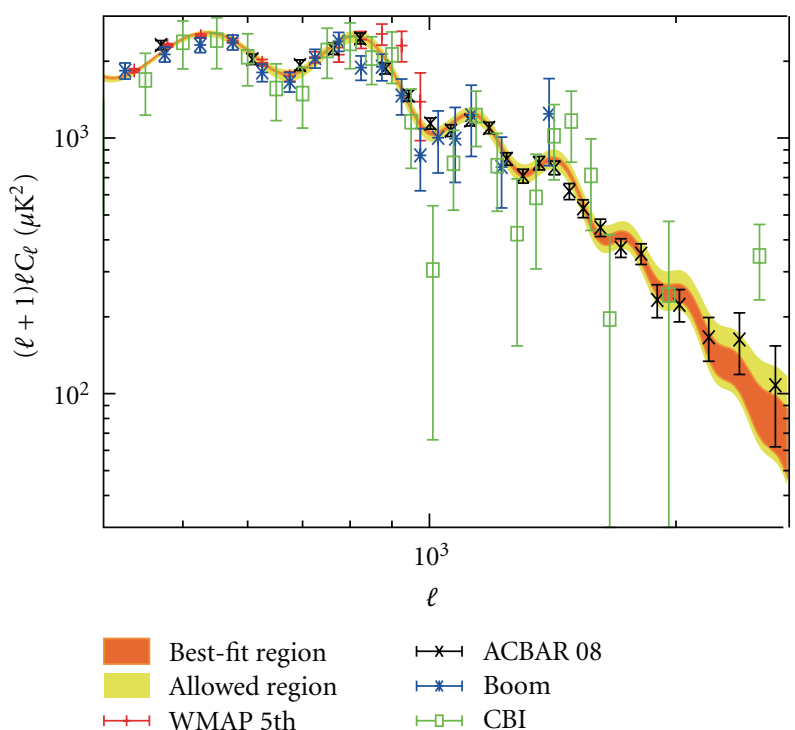

(b)

FIgURE 12: Comparison of the best-fit computed total power spectrum with the observed CMB spectra. Plots show various ranges for (a) TT $(2<\ell<2750)$ and (b) TT $(400<\ell<2750)$ modes. Orange regions are from the best-fit parameter set, and allowed regions are from constrained parameter set as Table 1. Red, black, blue, green, orange, and purple dots with error bars show WMAP 5 years, ACBAR 08, Boomerang, and CBI data, respectively. Since the SZ effect depends upon frequencies of the CMB for the TT mode, we plot the best fit and allowed regions in (a) and (b) surrounded by the curves with the SZ effect at the $K(22.8 \mathrm{GHz})$ band (upper curves) and without the SZ effect (lower curves).

for smaller $\ell<200$, while a PMF dominates this mode for $\ell>200$. Hence, if the gravity wave background without a PMF is generated by inflation, the origin of the gravity wave background on large scales will be inflation, while the background for smaller scales is from a PMF.

Based upon the current status of the field, we can summarize the following: (1) a PMF mainly affects the $\mathrm{CMB}$ temperature fluctuations and polarization anisotropies for small angular scales; (2) fits to observations of the CMB polarization anisotropies for small angular scales are generally better when effects of the PMF are included; (3) a PMF can either increase or decrease the matter density fluctuations on scales less than that of galaxy clusters.

6.1. Comparison with Other Determinations. As noted above, in our previous numerical estimate [29], the curvature perturbations of the scalar mode were too small to stabilize the numerical calculations for large scales and early times. This is caused by an unwanted cancellation between contributions from the primordial magnetic field and the primordial radiation fields which seemed to imply excess power for the lowest multipoles of the CMB. In order to solve this problem, we have adopted the numerical approach and initial conditions for the matter contributions from [79]. This formulation has been developed independently and gives consistent results for large scales and early times. We have now confirmed that our results are consistent not only with results of [79], but also those of other groups which employ seminumerical methods (e.g., [30]).
TABle 2: Effects of the PMF on each physical process.

\begin{tabular}{|c|c|c|}
\hline Topics & $\left|B_{\lambda}\right| \ll 1 \mathrm{nG}$ & $\left|B_{\lambda}\right| \sim 1 \mathrm{nG}$ \\
\hline The origin of PMF & After inflation & Inflation \\
\hline $\begin{array}{l}\text { The gravitational } \\
\text { wave background }\end{array}$ & Inflation & $\begin{array}{l}\text { Large-scale inflation: } \\
\text { Small-scale: PMF }\end{array}$ \\
\hline $\begin{array}{l}\text { The formation of } \\
\text { LSS }\end{array}$ & $\begin{array}{l}\text { Necessity of some } \\
\text { generation model for } \\
\text { the magnetic field or } \\
\text { vorticity }\end{array}$ & $\begin{array}{l}\text { Necessity for proper } \\
\text { initial conditions } \\
\text { considering the } \\
\text { magnetic field and } \\
\text { vorticity }\end{array}$ \\
\hline $\begin{array}{l}\text { The magnetic field } \\
\text { in clusters of } \\
\text { galaxies }\end{array}$ & $\begin{array}{l}\text { Necessity of some } \\
\text { global magnetic field } \\
\text { generation mode }+ \\
\text { amplification model }\end{array}$ & PMF origin \\
\hline $\begin{array}{l}\text { The reionization } \\
\text { and the first objects }\end{array}$ & & $\begin{array}{l}\text { Formation of the first } \\
\text { stars and the } \\
\text { reionization are } \\
\text { stimulated by a PMF }\end{array}$ \\
\hline
\end{tabular}

6.2. Influence of the PMF on Large-Scale Structure. In [38], we deduced the effects of a PMF on the matter energy density fields by considering a stochastic PMF that depends upon scale. We then quantitatively discussed the effect of a PMF on the seeds of LSS in the early universe. We have also considered more general effects of the PMF than those considered in previous work. For example, we included not only the magnetic field tension but also the increases in pressure and energy density perturbations from the 


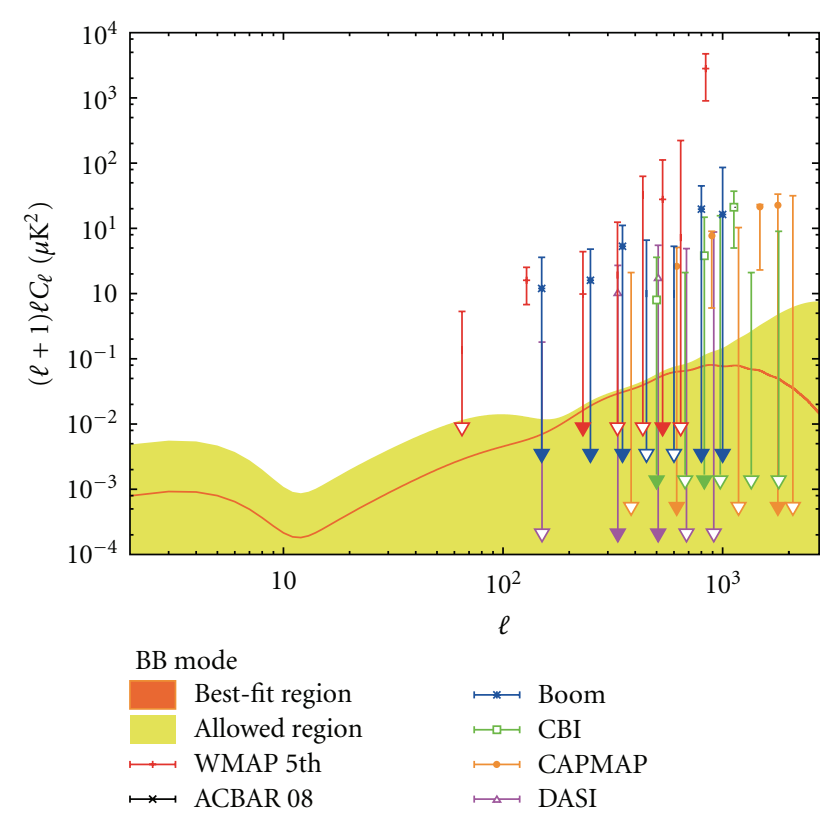

FIgURE 13: Comparison of the best-fit computed total power spectrum with the observed $\mathrm{CMB}$ spectra. The orange region is from the best-fit parameter set, and the allowed region is from the constrained parameter set as given in Table 1. Red, black, blue, green, orange, and purple dots with error bars show WMAP 5 years, ACBAR 08, Boomerang, CBI, CAPMAP, and DASI data, respectively. Downward arrows for the error bars on this figure indicate that the data points are upper limits. Since the BB mode is not affected by the SZ effect, the upper curves and lower curves in this figure are defined by the constrained cosmological parameters of Table 1 .

field. Furthermore, by considering the correlation between a PMF and the matter density fluctuations and utilizing mathematically exact stochastic PMF power spectrum sets, we have obtained reasonable and accurate models for the evolution of baryons, CDM, and photons and, therefore, the large scale structure. We have shown [38] that the PMF can play a very different role on the evolution of density perturbations depending upon how the PMF and matter fluctuations are correlated. We have also considered the fact that after decoupling, the CDM is influenced indirectly by the PMF through gravitational interaction.

We reported in [29] that a PMF on small angular scales $(\lambda \approx 1 \mathrm{Mpc})$ and a field strength of $B_{\lambda} \sim 1 \mathrm{nG}[15,17$, $18,26,27,80]$ provides a new interpretation for the excess of $\mathrm{CMB}$ anisotropies for high multipoles. We showed that if a PMF with such strength was present, it is very likely that it has affected the formation of large-scale structure. In [81] it was suggested that in order to avoid false coupling between the baryons and the CDM on small scales, one should use independent transfer functions for the baryons and CDM. A PMF would be another source of this difference in the transfer function for baryons and the CDM. Since the density perturbations in the early universe have evolved to the present LSS, the evolution of the LSS with a PMF becomes different from that without a PMF. We have shown that the baryon and CDM energy density perturbations evolve very differently in the presence of a PMF; and with a PMF taken into consideration, the evolution of large-scale structure becomes more complicated.

6.3. Future Observations. Magnetic fields affect the generation and evolution of a wide variety of cosmological and stellar objects in the universe, for example, galaxy clusters, galaxies, primordial stars, and so forth, (cf. Table 2). Therefore, it is important to constrain the parameters characterizing the PMF in order to study their effects on physical processes in the universe. In this regard, forthcoming missions, for example, QUIET, PolarBear, and Planck, are expected to provide much more precise data on the $\mathrm{CMB}$ temperature and polarization anisotropies. At the same time, data on the formation of large-scale structure will continue to be measured more precisely both by future space-based observations and from continuing groundbased observations such as the SDSS and 2dF projects.

As summarized in this paper, at the present time, we now understand the main effects of a PMF on both the CMB and the matter power spectrum. The important task remaining in the future will be to apply our new and correct numerical methods for calculating all PMF fluctuations of the scalar, vector, and tensor modes in the TT and $\mathrm{BB}$ power spectra (see Figures 11, 12, and 13) to fit the new observations as they emerge. Only through a critical comparison with such future data can we hope to ultimately clarify the magnitude and origin of the PMF in the early universe.

Although some degeneracy between the standard cosmological and PMF parameters is expected in the present data, we believe that by simultaneously investigating both the CMB anisotropies and matter power spectra one can resolve these degeneracies. The ultimate establishment of the existence and properties of a PMF will permit a better understanding of its generation and evolution and also provide new insight into the formation of LSS as well as a possible new probe of the physics of the early universe.

\section{Appendix}

\section{Initial Conditions}

In this appendix, we introduce the initial conditions of compensated magnetic modes with massless neutrinos in the synchronous gauge which were derived by [56]. Subscripts in this appendix indicate the standard cosmological species: $\gamma$ : photon, $v$ : massless neutrino, b: baryon, and CDM: cold dark matter. We define the density closure parameters as the ratios of density parameter as $\Omega_{x}=\rho_{x} / \rho_{\text {cr }}$, where $\rho_{\text {cr }}$ is the critical density of the universe and the subscript $x$ denotes the various species listed above. We also define ratios of $R_{\gamma}=$ $\Omega_{\gamma} / \Omega_{\mathrm{r}}, R_{\nu}=\Omega_{\gamma} / \Omega_{\mathrm{r}}$, and $R_{\mathrm{b}}=\Omega_{\mathrm{b}} / \Omega_{\mathrm{m}}$, where $\Omega_{\mathrm{r}}=\Omega_{\gamma}+\Omega_{\nu}$ and $\Omega_{\mathrm{m}}=\Omega_{\mathrm{CDM}}+\Omega_{\mathrm{b}}$.

\section{Scalar Mode.}

Potential

$$
h=-3 R_{\gamma} \frac{\beta}{\alpha} \tau+\frac{9}{2} R_{\gamma} \frac{\beta^{2}}{\alpha^{2}} \tau^{2}+\mathcal{O}\left(\tau^{3}\right) .
$$


Curvature potential

$$
\eta=\frac{1}{2} R_{\gamma} \frac{\beta}{\alpha} \tau+\left[\frac{R_{v} R_{\gamma}}{6\left(4 R_{v}+15\right)} k^{2}-\frac{3}{4} R_{\gamma} \frac{\beta^{2}}{\alpha^{2}}\right] \tau^{2}+\mathcal{O}\left(\tau^{3}\right) .
$$

Density perturbations

$$
\begin{aligned}
\delta_{\mathrm{CDM}}^{(S)} & =-\frac{3}{4} R_{\gamma}+\frac{3}{2} R_{\gamma} \frac{\beta}{\alpha} \tau-\frac{9}{4} R_{\gamma} \frac{\beta^{2}}{\alpha^{2}} \tau^{2}+\mathcal{O}\left(\tau^{3}\right), \\
\delta_{\gamma}^{(\mathrm{S})} & =-R_{\gamma}+2 R_{\gamma} \frac{\beta}{\alpha} \tau-\left(\frac{R_{v}}{6} k^{3}+3 R_{\gamma} \frac{\beta^{2}}{\alpha^{2}}\right) \tau^{2}+\mathcal{O}\left(\tau^{3}\right), \\
\delta_{\gamma}^{(\mathrm{S})} & =-R_{\gamma}+2 R_{\gamma} \frac{\beta}{\alpha} \tau+\left(\frac{R_{\gamma}}{6} k^{3}-3 R_{\gamma} \frac{\beta^{2}}{\alpha^{2}}\right) \tau^{2}+\mathcal{O}\left(\tau^{3}\right), \\
\delta_{\mathrm{b}}^{(\mathrm{S})} & =\frac{3}{4} \delta_{\gamma}^{(\mathrm{S})} .
\end{aligned}
$$

Velocity perturbations

$$
\begin{aligned}
& v_{\gamma}^{(\mathrm{S})}=\frac{1}{4} R_{\nu} k \tau+\frac{1}{4}\left(1-2 R_{\nu}-3 R_{\mathrm{b}} R_{\nu}+R_{\nu}^{2}\right) \frac{\beta}{\alpha} k \tau^{2}+\mathcal{O}\left(\tau^{3}\right), \\
& v_{\nu}^{(\mathrm{S})}=\frac{1}{4} R_{\gamma} k \tau+\frac{1}{4} R_{\gamma} \frac{\beta}{\alpha} k \tau^{2}+\mathcal{O}\left(\tau^{3}\right), \\
& v_{\mathrm{b}}^{(\mathrm{S})}=v_{\gamma}^{(S)}
\end{aligned}
$$

Anisotropic stress

$$
\Pi_{\nu}^{(\mathrm{S})}=-\frac{3 R_{\gamma}}{4 R_{v}+15} k^{2} \tau^{2}+\mathcal{O}\left(\tau^{3}\right) .
$$

Boltzmann hierarchy of neutrinos

$$
F_{\nu 3}^{(\mathrm{S})}=-\frac{R_{\gamma}}{4 R_{v}+15} k^{3} \tau^{3}+\mathcal{O}\left(\tau^{4}\right)
$$

Vector Mode.

Vector potential

$$
\sigma^{(\mathrm{V})}=\frac{15 R_{\gamma}}{56 R_{v}+210} k \tau-\frac{225 R_{\gamma}}{7\left(8 R_{v}^{2}+90 R_{v}+225\right)} \frac{\beta}{\alpha} k \tau^{2}+\mathcal{O}\left(\tau^{3}\right) .
$$

Velocity perturbations

$$
\begin{aligned}
& v_{\gamma}^{(\mathrm{V})}=-\frac{1}{8} k \tau+\frac{3 R_{\mathrm{b}}}{8 R_{\gamma}} \frac{\beta}{\alpha} k \tau^{2}+\mathcal{O}\left(\tau^{3}\right), \\
& v_{\nu}^{(\mathrm{V})}=-\frac{R_{\gamma}}{8 R_{\gamma}} k \tau+\mathcal{O}\left(\tau^{3}\right), \\
& v_{\mathrm{b}}^{(\mathrm{V})}=v_{\gamma}^{(\mathrm{V})} .
\end{aligned}
$$

Anisotropic stress

$\Pi_{\nu}^{(\mathrm{V})}=-\frac{R_{\gamma}}{R_{\nu}}+\frac{45 R_{\gamma}}{56 R_{v}^{2}+210 R_{v}} k^{2} \tau^{2}+\mathcal{O}\left(\tau^{3}\right)$

Boltzmann hierarchy of neutrinos

$$
F_{\nu 3}^{(\mathrm{V})}=-\sqrt{\frac{2}{3}} \frac{R_{\gamma}}{R_{v}} k \tau+\mathcal{O}\left(\tau^{3}\right) .
$$

Tensor Mode.

Tensor potential

$$
H^{(\mathrm{T})}=\frac{5 R_{\gamma}}{28\left(4 R_{v}+15\right)} k^{2} \tau^{2}+\mathcal{O}\left(\tau^{3}\right) .
$$

Anisotropic stress

$$
\Pi_{\nu}^{(\mathrm{T})}=-\frac{R_{\gamma}}{R_{v}}+\frac{15 R_{\gamma}}{56 R_{v}^{2}+210 R_{v}} k^{2} \tau^{2}+\mathcal{O}\left(\tau^{3}\right) .
$$

Boltzmann hierarchy of neutrinos

$$
F_{\nu 3}^{(\mathrm{T})}=-\frac{\sqrt{5}}{2} \frac{R_{\gamma}}{R_{v}} k \tau+\mathcal{O}\left(\tau^{3}\right) .
$$

\section{Acknowledgments}

This work has been supported in part by Grants-in-Aid for Scientific Research (nos. 20105004, 20244035, 21740177, and 20169444) of the Ministry of Education, Culture, Sports, Science and Technology of Japan. This work is also supported by the JSPS Core-to-Core Program, International Research Network for Exotic Femto Systems (EFES). Work at UND was supported in part by the US Department of Energy under research Grant no.DE-FG02-95-ER40934.

\section{References}

[1] A. M. Wolfe, K. M. Lanzetta, and A. L. Oren, "Magnetic fields in damped Ly $\alpha$ systems," Astrophysical Journal, vol. 388, no. 1, pp. 17-22, 1992.

[2] T. E. Clarke, P. P. Kronberg, and H. Böhringer, "A new radioX-ray probe of galaxy cluster magnetic fields," Astrophysical Journal, vol. 547, no. 2, pp. L111-L114, 2001.

[3] Y. Xu, P. P. Kronberg, S. Habib, and Q. W. Dufton, "A faraday rotation search for magnetic fields in large-scale structure," Astrophysical Journal, vol. 637, no. 1, pp. 19-26, 2006.

[4] M. S. Turner and L. M. Widrow, "Inflation-produced, largescale magnetic fields," Physical Review D, vol. 37, no. 10, pp. 2743-2754, 1988.

[5] B. Ratra, "Cosmological "seed" magnetic field from inflation," Astrophysical Journal, vol. 391, no. 1, pp. L1-L4, 1992.

[6] K. Bamba and J. Yokoyama, "Large-scale magnetic fields from dilaton inflation in noncommutative spacetime," Physical Review D, vol. 70, no. 8, Article ID 083508, 2004.

[7] T. Vachaspati, "Magnetic fields from cosmological phase transitions," Physics Letters B, vol. 265, no. 3-4, pp. 258-261, 1991. 
[8] T. W. B. Kibble and A. Vilenkin, "Phase equilibration in bubble collisions," Physical Review D, vol. 52, no. 2, pp. 679-688, 1995.

[9] J. Ahonen and K. Enqvist, "Magnetic field generation in first order phase transition bubble collisions," Physical Review D, vol. 57, no. 2, pp. 664-673, 1998.

[10] M. Joyce and M. Shaposhnikov, "Primordial magnetic fields, right electrons, and the Abelian anomaly," Physical Review Letters, vol. 79, no. 7, pp. 1193-1196, 1997.

[11] K. Takahashi, K. Ichiki, H. Ohno, and H. Hanayama, "Magnetic field generation from cosmological perturbations," Physical Review Letters, vol. 95, no. 12, Article ID 121301, 4 pages, 2005.

[12] H. Hanayama, K. Takahashi, K. Kotake, M. Oguri, K. Ichiki, and $\mathrm{H}$. Ohno, "Biermann mechanism in primordial supernova remnant and seed magnetic fields," Astrophysical Journal, vol. 633, no. 2, pp. 941-945, 2005.

[13] K. Ichiki, K. Takahashi, H. Ohno, H. Hanayama, and N. Sugiyama, "Cosmological magnetic field: a fossil of density perturbations in the early universe," Science, vol. 311, no. 5762, pp. 827-829, 2006.

[14] K. Subramanian and J. D. Barrow, "Microwave background signals from tangled magnetic fields," Physical Review Letters, vol. 81, no. 17, pp. 3575-3578, 1998.

[15] A. Mack, T. Kahniashvili, and A. Kosowsky, "Microwave background signatures of a primordial stochastic magnetic field," Physical Review D, vol. 65, no. 12, Article ID 123004, 2002.

[16] K. Subramanian and J. D. Barrow, "Small-scale microwave background anisotropies arising from tangled primordial magnetic fields," Monthly Notices of the Royal Astronomical Society, vol. 335, no. 3, pp. L57-L61, 2002.

[17] A. Lewis, "CMB anisotropies from primordial inhomogeneous magnetic fields," Physical Review D, vol. 70, no. 4, Article ID 043011, 4 pages, 2004.

[18] D. G. Yamazaki, K. Ichiki, and T. Kajino, "Constraining the primordial magnetic field from cosmic microwave background anisotropies at higher multipoles," Astrophysical Journal, vol. 625, no. 1, pp. L1-L4, 2005.

[19] T. Kahniashvili and B. Ratra, "Effects of cosmological magnetic helicity on the cosmic microwave background," Physical Review D, vol. 71, no. 10, Article ID 103006, 2005.

[20] A. Challinor, "Cosmic microwave background polarization analysis," in Data Analysis in Cosmology, vol. 653 of Lecture Notes in Physics, pp. 71-104, Springer, Berlin, Germany, 2004.

[21] A. D. Dolgov, "Cosmological magnetic fields and CMBR polarization," http://arxiv.org/abs/astro-ph/0503447.

[22] R. Gopal and S. K. Sethi, "Tangled magnetic fields and CMBR signal from reionization epoch," Physical Review D, vol. 72, no. 10, Article ID 103003, 12 pages, 2005.

[23] D. G. Yamazaki, K. Ichiki, and T. Kajino, "Primordial magnetic field at the photon last scattering surface," Nuclear Physics A, vol. 758, no. 1-4, pp. 791-794, 2005.

[24] T. Kahniashvili and B. Ratra, "CMB anisotropies due to cosmological magnetosonic waves," Physical Review D, vol. 75, no. 2, Article ID 023002, 2007.

[25] D. G. Yamazaki, K. Ichiki, K. I. Umezu, and H. Hanayama, "Effect of primordial magnetic field on seeds for large scale structure," Physical Review D, vol. 74, no. 12, Article ID 123518, 2006.

[26] D. G. Yamazaki, K. Ichiki, T. Kajino, and G. J. Mathews, "Constraints on the evolution of the primordial magnetic field from the small-scale cosmic microwave background angular anisotropy," Astrophysical Journal, vol. 646, no. 2, pp. 719-729, 2006.
[27] D. G. Yamazaki, K. Ichiki, T. Kajino, and G. J. Mathews, "Primordial magnetic field constrained from $\mathrm{CMB}$ anisotropies, and its generation and evolution before, during and after the BBN," in Proceedings of the 9th Symposium on Nuclei in the Cosmos (PoSNIC '06), pp. 194-199, June 2006.

[28] M. Giovannini, "Tight coupling expansion and fully inhomogeneous magnetic fields," Physical Review D, vol. 74, no. 6, Article ID 063002, 2006.

[29] D. G. Yamazaki, K. Ichiki, T. Kajino, and G. J. Mathews, "Effects of a primordial magnetic field on low and high multipoles of the cosmic microwave background," Physical Review D, vol. 77, no. 4, Article ID 043005, 2008.

[30] D. Paoletti, F. Finelli, and F. Paci, "The scalar, vector and tensor contributions of a stochastic background of magnetic fields to cosmic microwave background anisotropies," Monthly Notices of the Royal Astronomical Society, vol. 396, no. 1, pp. 523-534, 2009.

[31] F. Finelli, F. Paci, and D. Paoletti, "Impact of stochastic primordial magnetic fields on the scalar contribution to cosmic microwave background anisotropies," Physical Review D, vol. 78, no. 2, Article ID 023510, 14 pages, 2008.

[32] D. G. Yamazaki, K. Ichiki, T. Kajino, and G. J. Mathews, "Constraints on the primordial magnetic field from $\sigma 8$," Physical Review D, vol. 78, no. 12, Article ID 123001, 2008.

[33] D. G. Yamazaki, K. Ichiki, T. Kajino, and G. J. Mathews, "A strong constraint on the neutrino mass from the formation of large scale structure in the presence of the primordial magnetic field," in Proceedings of the 10th Symposium on Nuclei in the Cosmos (PoSNIC '08), p. 239, July-August 2008.

[34] S. K. Sethi, B. B. Nath, and K. Subramanian, "Primordial magnetic fields and formation of molecular hydrogen," Monthly Notices of the Royal Astronomical Society, vol. 387, no. 4, pp. 1589-1596, 2008.

[35] K. Kojima, K. Ichiki, D. G. Yamazaki, T. Kajino, and G. J. Mathews, "Neutrino mass effects on vector and tensor CMB anisotropies in the presence of a primordial magnetic field," Physical Review D, vol. 78, no. 4, Article ID 045010, 2008.

[36] T. Kahniashvili, G. Lavrelashvili, and B. Ratra, "CMB temperature anisotropy from broken spatial isotropy due to a homogeneous cosmological magnetic field," Physical Review $D$, vol. 78, no. 6, Article ID 063012, 2008.

[37] M. Giovannini and K. E. Kunze, "Faraday rotation, stochastic magnetic fields, and CMB maps," Physical Review D, vol. 78, no. 2, Article ID 023010, 2008.

[38] D. G. Yamazaki, K. Ichiki, T. Kajino, and G. J. Mathews, "New constraints on the primordial magnetic field," Physical Review D, vol. 81, no. 2, Article ID 023008, 2010.

[39] D. G. Yamazaki, K. Ichiki, T. Kajino, and G. J. Mathews, "Constraints on the neutrino mass and the primordial magnetic field from the matter density fluctuation parameter $\sigma 8$," Physical Review D, vol. 81, no. 10, Article ID 103519, 2010.

[40] D. G. Yamazaki, K. Ichiki, T. Kajino, and G. J. Mathews, "Magnetic field effects on the CMB and large-scale structure," in Proceedings of the 10th International Symposium on Origin of Matter and Evolution of Galaxies (OMEG '10), vol. 1269 of AIP Conf. Proc., pp. 57-62, August 2010.

[41] I. Brown and R. Crittenden, "Non-Gaussianity from cosmic magnetic fields," Physical Review D, vol. 72, no. 6, Article ID 063002, 15 pages, 2005.

[42] T. R. Seshadri and K. Subramanian, "Cosmic microwave background bispectrum from primordial magnetic fields on large angular scales," Physical Review Letters, vol. 103, no. 8, Article ID 081303, 2009. 
[43] C. Caprini, F. Finelli, D. Paoletti, and A. Riotto, "The cosmic microwave background temperature bispectrum from scalar perturbations induced by primordial magnetic fields," Journal of Cosmology and Astroparticle Physics, vol. 2009, no. 6, article 021, 2009.

[44] A. Kosowsky and A. Loeb, "Faraday rotation of microwave background polarization by a primordial magnetic field," Astrophysical Journal, vol. 469, no. 1, pp. 1-6, 1996.

[45] T. Kolatt, "Determination of the primordial magnetic field power spectrum by faraday rotation correlations," Astrophysical Journal, vol. 495, no. 2, pp. 564-579, 1998.

[46] A. Kosowsky, T. Kahniashvili, G. Lavrelashvili, and B. Ratra, "Faraday rotation of the cosmic microwave background polarization by a stochastic magnetic field," Physical Review D, vol. 71, no. 4, Article ID 043006, 2005.

[47] L. Campanelli, A. D. Dolgov, M. Giannotti, and F. L. Villante, "Faraday rotation of the cosmic microwave background polarization and primordial magnetic field properties," Astrophysical Journal, vol. 616, no. 1, pp. 1-7, 2004.

[48] G. Chen, P. Mukherjee, T. Kahniashvili, B. Ratra, and Y. Wang, "Looking for cosmological Alfvén waves in Wilkinson Microwave Anisotropy Probe data," Astrophysical Journal, vol. 611, no. 2, pp. 655-659, 2004.

[49] A. Bernui and W. S. Hipólito-Ricaldi, "Can a primordial magnetic field originate large-scale anomalies in WMAP data?" Monthly Notices of the Royal Astronomical Society, vol. 389, no. 3, pp. 1453-1460, 2008.

[50] S. K. Sethi, "Large-scale magnetic fields: galaxy two-point correlation function," Monthly Notices of the Royal Astronomical Society, vol. 342, no. 3, pp. 961-970, 2003.

[51] S. K. Sethi and K. Subramanian, "Primordial magnetic fields in the post-recombination era and early reionization," Monthly Notices of the Royal Astronomical Society, vol. 356, no. 2, pp. 778-788, 2005.

[52] K. Jedamzik, V. Katalinić, and A. V. Olinto, "Damping of cosmic magnetic fields," Physical Review D, vol. 57, no. 6, pp. 3264-3284, 1998.

[53] K. Subramanian and J. D. Barrow, "Magnetohydrodynamics in the early universe and the damping of nonlinear Alfvén waves," Physical Review D, vol. 58, no. 8, Article ID 083502, 8 pages, 1998.

[54] R. Banerjee and K. Jedamzik, "Evolution of cosmic magnetic fields: from the very early universe, to recombination, to the present," Physical Review D, vol. 70, no. 12, Article ID 123003, 25 pages, 2004.

[55] A. Bûcher, K. Moodley, and N. Turok, "General primordial cosmic perturbatio," Physical Review D, vol. 62, no. 8, Article ID 083508, 10 pages, 2000.

[56] J. R. Shaw and A. Lewis, "Massive neutrinos and magnetic fields in the early universe," Physical Review D, vol. 81, no. 4, Article ID 043517, 2010.

[57] K. Yamamoto, N. Sugiyama, and H. Sato, "Evolution of smallscale cosmological baryon perturbations and matter transfer functions," Astrophysical Journal, vol. 501, no. 2, pp. 442-460, 1998.

[58] T. R. Seshadri and K. Subramanian, "Cosmic microwave background polarization signals from tangled magnetic fields," Physical Review Letters, vol. 87, no. 10, Article ID 101301, 4 pages, 2001.

[59] A. A. Starobinskii, "Spectrum of relict gravitational radiation and the early state of the universe," ZHETF Pis'ma v Redaktsiiu, vol. 30, pp. 719-723, 1979.
[60] V. A. Rubakov, M. V. Sazhin, and A. V. Veryaskin, "Graviton creation in the inflationary universe and the grand unification scale," Physics Letters B, vol. 115, no. 3, pp. 189-192, 1982.

[61] Z. Kraicheva, V. Stanishev, V. Genkov, and L. Iliev, "TT Arietis: 1985-1999 accretion disc behaviour," Astronomy and Astrophysics, vol. 351, no. 2, pp. 607-618, 1999.

[62] J. R. Pritchard and M. Kamionkowski, "Cosmic microwave background fluctuations from gravitational waves: an analytic approach," Annals of Physics, vol. 318, no. 1, pp. 2-36, 2005.

[63] P. J. E. Peebles, The Large-Scale Structure of the Universe, Princeton University Press, Princeton, NJ, USA, 1980.

[64] D. N. Spergel, R. Bean, O. Doré et al., "Three-year Wilkinson Microwave Anisotropy Probe (WMAP) observations: implications for cosmology," The Astrophysical Journal Supplement Series, vol. 170, no. 2, p. 377, 2007.

[65] G. Hinshaw, M. R. Nolta, C. L. Bennett et al., "Three-year Wilkinson Microwave Anisotropy Probe (WMAP) observations: temperature analysis," The Astrophysical Journal Supplement Series, vol. 170, no. 2, p. 288, 2007.

[66] L. Page, G. Hinshaw, E. Komatsu et al., “Three-year Wilkinson Microwave Anisotropy Probe (WMAP) observations: polarization analysis," The Astrophysical Journal Supplement Series, vol. 170, no. 2, p. 335, 2007.

[67] A. Lewis and S. Bridle, "Cosmological parameters from CMB and other data: a Monte Carlo approach," Physical Review D, vol. 66, no. 10, Article ID 103511, 2002.

[68] S. Cole, W. J. Percival, J. A. Peacock et al., "The 2dF Galaxy Redshift survey: power-spectrum analysis of the final data set and cosmological implications," Monthly Notices of the Royal Astronomical Society, vol. 362, no. 2, pp. 505-534, 2005.

[69] M. Tegmark, D. J. Eisenstein, M. A. Strauss et al., "Cosmological constraints from the SDSS luminous red galaxies," Physical Review D, vol. 74, no. 12, Article ID 123507, 2006.

[70] E. Rozo, R. H. Wechsler, B. P. Koester et al., "Cosmological constraints from SDSS maxBCG cluster abundances," Astrophysical Journal, vol. 708, pp. 645-660, 2010.

[71] A. J. Ross, R. J. Brunner, and A. D. Myers, "Normalization of the matter power spectrum via higher order angular correlations of luminous red galaxies," Astrophysical Journal, vol. 682, no. 2, pp. 737-744, 2008.

[72] J. Dunkley, E. Komatsu, M. R. Nolta et al., "Five-year wilkinson microwave anisotropy probe observations: likelihoods and parameters from the WMAP data," Astrophysical Journal, Supplement Series, vol. 180, no. 2, pp. 306-329, 2009.

[73] G. Hinshaw, J. L. Weiland, R. S. Hill et al., "Five-year wilkinson microwave anisotropy probe observations: data processing, sky maps and basic results," The Astrophysical Journal Supplement Series, vol. 180, no. 2, pp. 225-245, 2009.

[74] C. L. Kuo, P. A. R. Ade, J. J. Bock et al., "Improved measurements of the CMB power spectrum with ACBAR," Astrophysical Journal, vol. 664, no. 2, pp. 687-701, 2007.

[75] J. L. Sievers, C. Achermann, J. R. Bond et al., "Implications of the cosmic background imager polarization data," Astrophysical Journal, vol. 660, no. 2, pp. 976-987, 2007.

[76] W. C. Jones, P. A. R. Ade, J. J. Bock et al., "A measurement of the angular power spectrum of the CMB temperature anisotropy from the 2003 flight of Boomerang," Astrophysical Journal, vol. 647, no. 2, pp. 823-832, 2006.

[77] C. Caprini and R. Durrer, "Gravitational wave production: a strong constraint on primordial magnetic fields," Physical Review D, vol. 65, no. 2, Article ID 023517, 2002. 
[78] R. Durrer and C. Caprini, "Primordial magnetic fields and causality," Journal of Cosmology and Astroparticle Physics, no. 11, pp. 171-178, 2003.

[79] K. Kojima and K. Ichiki, "Evolution of cosmological perturbations in the presence of primordial magnetic fields," http://arxiv.org/abs/0902.1367.

[80] R. Durrer, P. G. Ferreira, and T. Kahniashvili, "Tensor microwave anisotropies from a stochastic magnetic field," Physical Review D, vol. 61, no. 4, Article ID 043001, 6 pages, 2000.

[81] N. Yoshida, N. Sugiyama, and L. Hernquist, "The evolution of ban on density fluctuations in multicomponent cosmological simulations," Monthly Notices of the Royal Astronomical Society, vol. 344, no. 2, pp. 481-491, 2003. 

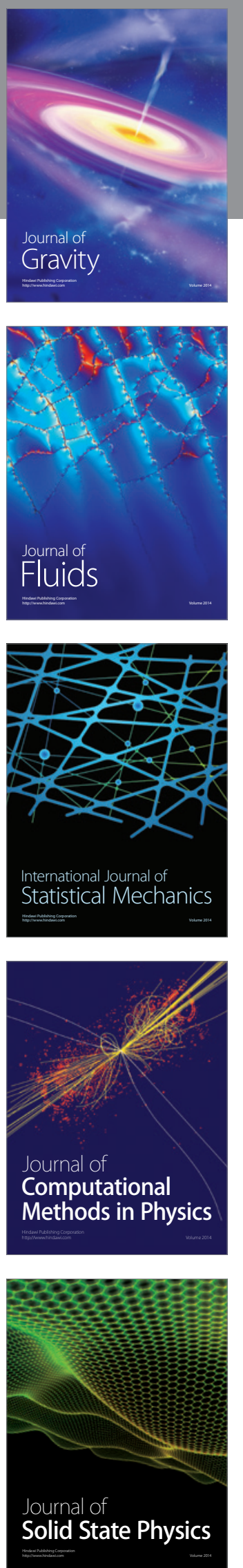

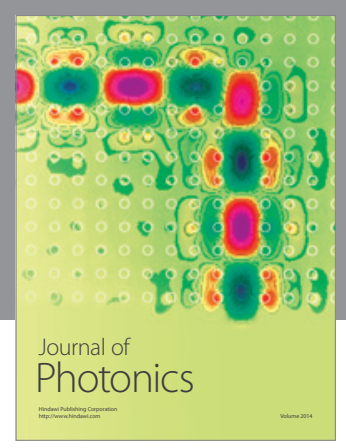

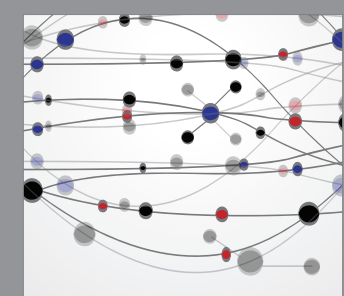

The Scientific World Journal
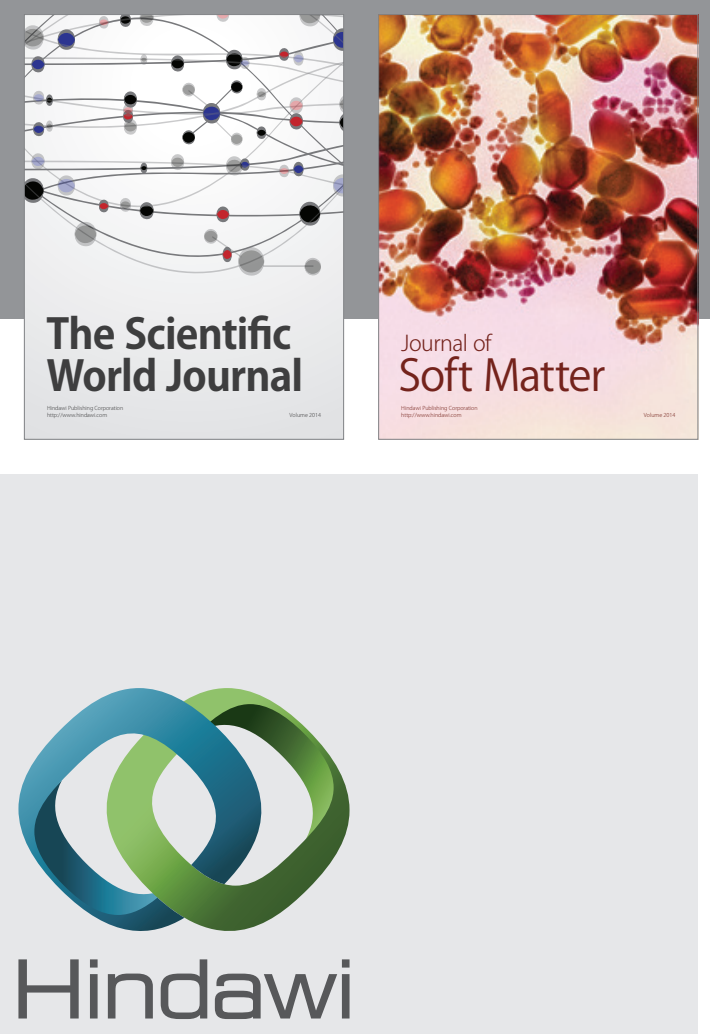

Submit your manuscripts at

http://www.hindawi.com
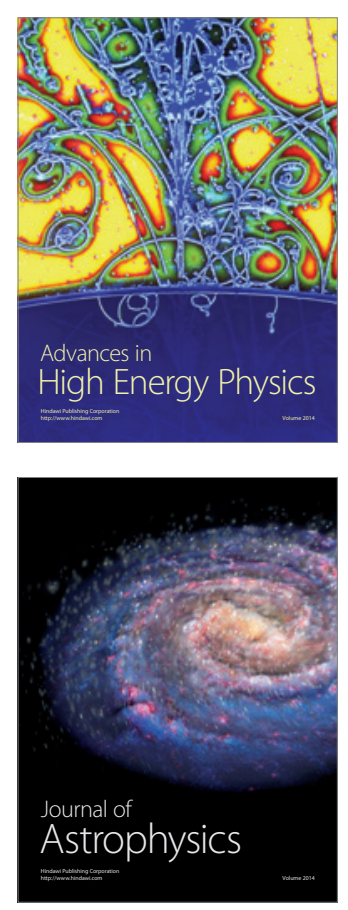
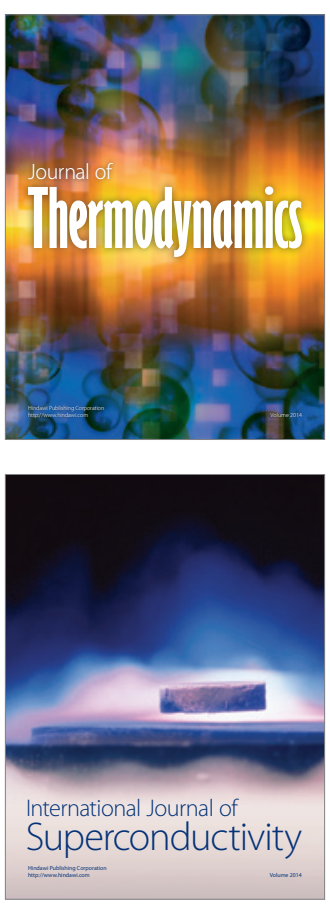
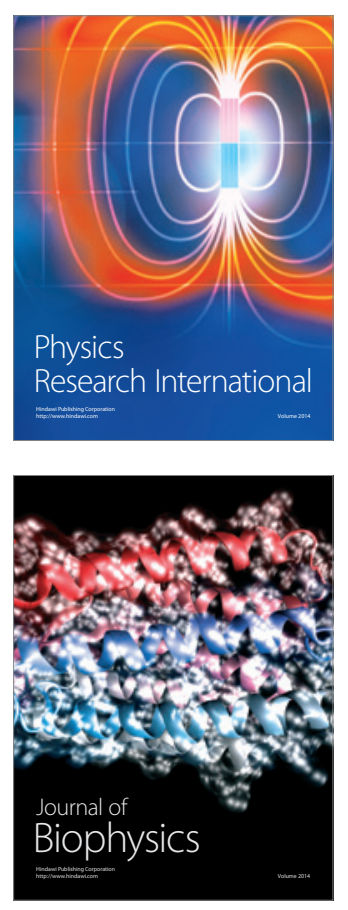
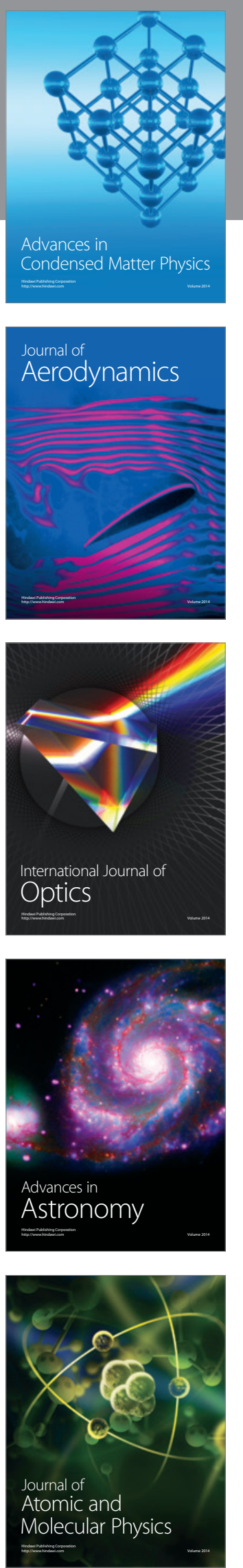